\title{
Diversity and Biocontrol Potential of Culturable Endophytic Fungi in Cotton
}

\author{
Lirong Jin ${ }^{1,2,3}$, Long Yang ${ }^{1}$, Wenjing $L i^{2,3}$, Dong $X u^{2,3}$, Nina Yang ${ }^{2,3}$, Guoqing $L^{1}{ }^{1}$ and \\ Peng Wan ${ }^{2,3 *}$
}

${ }^{1}$ State Key Laboratory of Agricultural Microbiology, Key Laboratory of Plant Pathology of Hubei Province, Huazhong Agricultural University, Wuhan, China, ${ }^{2}$ Key Laboratory of Integrated Pest Management Crops in Central China, Ministry of Agriculture, Hubei Academy of Agricultural Sciences, Wuhan, China, ${ }^{3}$ Hubei Key Laboratory of Crop Disease, Insect Pests and Weeds Control, Plant Protection, Soil and Fertilizer Research Institute, Hubei Academy of Agricultural Sciences, Wuhan, China

\section{OPEN ACCESS}

Edited by:

Palanivel Velmurugan,

Alagappa University, India

Reviewed by:

Masoomeh Shams-Ghahfarokhi,

Tarbiat Modares University, Iran

Muneer Ahmed Qazi,

Shah Abdul Latif University, Pakistan

${ }^{*}$ Correspondence:

Peng Wan

wanpenghb@126.com

Specialty section:

This article was submitted to Microbe and Virus Interactions with

a section of the journal Frontiers in Microbiology

Received: 22 April 2021

Accepted: 19 July 2021

Published: 13 August 2021

Citation:

Jin L, Yang L, Li W, Xu D, Yang $N$ Li GQ and Wan P (2021) Diversity and Biocontrol Potential of Culturable

Endophytic Fungi in Cotton.

Front. Microbiol. 12:698930.

doi: 10.3389/fmicb.2021.698930
Healthy cotton samples were collected and 93 endophytic fungal strains were isolated: 23 strains from the roots and 70 strains from the stems. Morphological characterization and ITS sequence analysis were used for the identification of these isolates. The results showed that the 93 strains including 20 species were highly diverse in terms of their taxonomy. Simpson's and Shannon's diversity indices were 0.915 and 3.848, respectively. Fusarium and Alternaria were the two dominant genera, constituting 19.4\% of the total strains. Then, 72 spore-producing strains were tested for the suppression of cotton Verticillium wilt (CWW) caused by Verticillium dahliae in a greenhouse. Five strains exhibited effective suppression of CVW with average efficacy values higher than $50 \%$. One of the effective strains, namely, Fusarium proliferatum 10R-7, was selected for the investigation of the role of fusaric acid, a secondary metabolite of strain 10R-7, in the suppression of $V$. dahliae and CWW. The results showed that $F$. proliferatum 10R7 could produce fusaric acid, and this metabolite exhibited $100 \%$ inhibition of mycelial growth of $V$. dahliae at concentrations higher than $20 \mu \mathrm{g} / \mathrm{ml}$. However, fusaric acid at 2.5 to $80 \mu \mathrm{g} / \mathrm{ml}$ was not effective in the suppression of CVW, compared with the control treatment with $V$. dahliae alone. F. proliferatum 10R-7 was labeled with green fluorescent protein (GFP), and the GFP-tagged strain was found to be able to colonize inside the taproots of cotton, suggesting that F. proliferatum 10R-7 is a true endophyte of cotton and endophytic colonization may play a role in the suppression of infection of cotton by V. dahliae.

Keywords: cotton, Verticillium dahliae, endophytic fungi, Fusarium proliferatum 10R-7, biological control

\section{INTRODUCTION}

Cotton is an economically important crop grown worldwide. Cotton Verticillium wilt caused by Verticillium dahliae Kleb. is a plant disease that leads to severe economic losses in cotton (Ma et al., 2002). As a soilborne plant disease, it is difficult to control it utilizing conventional fungicide (Berg et al., 2001). Meanwhile, $V$. dahliae could produce dormant structures called microsclerotia with high stress resistance. The microsclerotia can persist in soil for over 14 years (Klosterman et al., 2009), which increase the difficulty of controlling the disease. The method of controlling the disease includes breeding of resistant cultivars, change of 
agricultural planting pattern, and chemical control (Aguado et al., 2008; Erdogan and Benlioglu, 2010). However, these methods are not so effective or not environmentally friendly as expected (Erdogan and Benlioglu, 2010).

Biological control is one of the most promising approaches for suppressing Verticillium wilt because of its being environmentally friendly. Some fungi and bacteria have been reported as biocontrol agents (BCAs) against $V$. dahliae (Berg et al., 2001, 2005; Mercado-Blanco et al., 2004; Malandraki et al., 2008; Uppal et al., 2008; Erdogan and Benlioglu, 2010; Zheng et al., 2011; Deketelaere et al., 2017). There were also numerous reports about the biocontrol potential of Fusarium spp. to suppress V. dahliae (Deketelaere et al., 2017). These Fusarium spp. include F. culmorum (Dutta, 1981), F. lateritium (García et al., 2011), F. moniliforme (Varo et al., 2016a,b), F. solani (Zheng et al., 2011), and F. oxysporum (Díaz et al., 2005; Malandraki et al., 2008; Pantelides et al., 2009; Gizi et al., 2011; Veloso and Díaz, 2012; Angelopoulou et al., 2014; Zhang et al., 2015). Their primary mechanisms of action against $V$. dahliae are antibiosis (Dutta, 1981; García et al., 2011), competition for nutrients and/or ecological sites (Larkin and Fravel, 1999; Pantelides et al., 2009; Varo et al., 2016a,b), triggering of induced systemic resistance (Díaz et al., 2005; Olivain et al., 2006; Malandraki et al., 2008; Angelopoulou et al., 2014), and mycoparasitism (Nagtzaam et al., 1998). Competition included competition for nutrients and ecological sites on the root (Gizi et al., 2011; Angelopoulou et al., 2014).

Endophytic fungi are distributed widely and have been found in almost all of the plants. They are highly diversified in species composition across many plants (Rodriguez et al., 2009; Potshangbam et al., 2017). Previous studies have confirmed the diversity of cotton endophytic fungi (Ek-Ramos et al., 2013; Zhi-Fang Li et al., 2014). As a potential biocontrol resource, many endophytic fungi have the function of suppressing plant disease and insect pests, promoting growth of their host plants, and enhancing plant resistance (Backman and Sikora, 2008; Hassani et al., 2018). In the present study, we isolated 93 endophytic fungi from cotton, analyzed the diversity of the endophytic fungi, and evaluated the ability of these endophytic fungi to control cotton Verticillium wilt (CVW). The specific objectives were $(i)$ to isolate cotton endophytic fungi and analyze the diversity of the endophytic fungi, (ii) to screen endophytic fungi with high biocontrol efficacy against $V$. dahliae, and (iii) to investigate the possible biocontrol mechanisms of Fusarium proliferatum 10R-7 against $V$. dahliae by identifying the antifungal metabolites of $F$. proliferatum $10 \mathrm{R}-7$ and assessing its colonization in cotton roots.

\section{MATERIALS AND METHODS}

\section{Isolation of Endophytic Fungi}

One hundred and eighty healthy cotton samples were collected in the fields of Wuhan City $\left(30^{\circ} 28^{\prime} \mathrm{N}, 114^{\circ} 21^{\prime} \mathrm{E}\right)$, Hubei Province, China. First, the plant samples were rinsed with tap water and dried with filter paper. The samples were cut into small pieces (3-cm-long segments) and surface-sterilized by submerging in
$70 \%$ ethanol (v/v) for $1 \mathrm{~min}, 5 \%$ sodium hypochlorite (v/v) for $5 \mathrm{~min}$, and $70 \%$ ethanol again for $30 \mathrm{~s}$, followed by rinsing in autoclaved distilled water five times (Hallmann et al., 2006; Zhang et al., 2014). Then, the cotton tissues were blotted with sterilized filter paper and cut into short pieces with sterile scissors, placed in an autoclaved mortar containing $1 \mathrm{ml}$ of sterile water, and triturated with an autoclaved pestle. The mixtures were plated on aPDA (PDA amended with lactic acid, $\mathrm{pH}$ 2.5-3.5) media and were cultured at $25^{\circ} \mathrm{C}$ for approximately 2 weeks until the fungal endophyte hyphae grew. The fungal colonies were individually transferred to new PDA dishes, with one colony per dish, and incubated at $25^{\circ} \mathrm{C}$. Finally, the fungal cultures were purified and stored at $-80^{\circ} \mathrm{C}$.

\section{Identification of the Endophytic Fungi}

The endophytic fungi were identified by adopting the combined method of morphological and molecular identification. The morphological characteristics of the colonies and spores of the fungal isolates were examined (Kirk et al., 2008). Moreover, the internal transcribed spacer (ITS) region of rDNA (ITS1-5.8S rDNA-ITS2) of each isolate was amplified using the universal primers ITS1 (5'-TCC GTA GGT GAA CCT GCG G-3') and ITS4 (5'-TCC TCC GCT TAT TGA TAT GC-3'), and the amplified products were purified, cloned, and sequenced at Sangon Bioengineering Co., Ltd., Shanghai, China (Zhang et al., 2014). The BLAST program was used to compare the resulting sequence with that of the closest related fungal species in the NCBI database. Then, the strains were identified to species by consideration of the ITS sequence and the morphological characteristics.

Several indicators for diversity assessment, including species richness $(S)$, species proportion $(f)$, Simpson's diversity index $(D)$, and Shannon's diversity index $\left(H^{\prime}\right)$, were used to evaluate the community diversity of the endophytic isolates from cotton. The formulas are as follows:

$$
\begin{gathered}
P i=\frac{n i}{N} \\
D=1-\Sigma(P i)^{2} \\
H^{\prime}=-\Sigma P i \times \log _{2} P i
\end{gathered}
$$

where $n_{i}$ represents the number of isolates of the same species and $N$ represents the total number of endophytic fungi.

\section{Screening of Biocontrol Agents in a Greenhouse}

In the first round of screening, the fungi that produced spores were screened out by microscopic observation. The sporeproducing isolates were further screened based on the greenhouse experiments. The method was as follows: the seeds of upland cotton (Gossypium hirsutum) variety "JI11," a susceptible cultivar, were soaked in sterile water for $12 \mathrm{~h}$ and planted into nutrient vermiculite culture mixture (nursery substrate:sand $=1: 1, \mathrm{v} / \mathrm{v}$ ). When the seedlings grew to the two-true-leaf stage, the roots 
were uprooted and rinsed clean under tap water. The conidia of each endophytic fungus (EF) were collected from 7-dayold potato dextrose broth shake cultures and filtered through four layers of cheesecloth, followed by centrifugation to remove the supernatant. Then, the conidial precipitate was dissolved in sterile water and diluted to $1 \times 10^{7}$ conidia $/ \mathrm{ml}$. The conidia of $V$. dahliae V991b were harvested by washing 15-day-old PDA cultures (at $25^{\circ} \mathrm{C}$ ) with autoclaved distilled water, and the mixtures were filtered to remove the hyphae. Then, the conidial concentration was adjusted to $1 \times 10^{7}$ conidia $/ \mathrm{ml}$ for $V$. dahliae $(V d)$ and the endophytic fungus (EF). There were four treatments in this experiment: $\mathrm{EF}+V d, V d$ alone, $\mathrm{EF}$ alone, and sterile water control (CK). In the treatment of $\mathrm{EF}+V d$, the conidial suspensions of $V$. dahliae and the endophytic fungus were mixed in equal volumes to a final concentration of $5 \times 10^{6}$ conidia/ml. In the treatments of EF alone and $V d$ alone, the conidial suspensions of EF and $V d$ were separately diluted by half with sterile water. The roots of cotton seedlings (10-15 seedlings per treatment) were dipped for $20 \mathrm{~min}$ in the conidial suspensions of the four treatments, and each treatment included 10-15 seedlings. Then, the seedlings were transplanted into nutrient vermiculite culture mixtures in plastic pots $(12 \times 10 \mathrm{~cm}$, diameter $\times$ height) with three to five seedlings per pot for each treatment. The pots with the seedlings were maintained in a greenhouse $\left(25 \pm 3^{\circ} \mathrm{C}\right)$, and the seedlings were watered to maintain an appropriate humidity. At 15, 20, 25, and 30 days postinoculation (dpi), the seedlings were individually rated for severity of Verticillium wilt. Disease severity for each seedling was rated using a numeric scale of 0 to 4 , with $0=$ healthy; $1=$ one to two cotyledons showing wilting symptoms, $2=$ one true leaf showing wilting symptoms, 3 = two or more true leaves showing wilting symptoms, and $4=$ plant dead (Shi et al., 1993). The disease index $(D I)$ for each treatment was calculated on the basis of the disease severity rating and the number of cotton seedlings according to the following formula:

$$
D I=\frac{0 \times n 0+1 \times n 1+2 \times n 2+3 \times n 3+4 \times n 4}{N \times 4} \times 100
$$

where $n 0, n 1, n 2, n 3$, and $n 4$ represent the numbers of cotton seedlings with disease severity scores of $0,1,2,3$, and 4 , respectively, and the number $N$ represents the total number of tested cotton seedlings for each treatment. The biocontrol efficacy $(B E)$ was calculated according to the following formula:

$$
B E=[(D I \text { in control }-D I \text { in } E F) / D I \text { in control }] \times 100 \%
$$

This primary screening experiment was carried out three times, and the fungal isolates screened in the primary screening were subjected to the secondary screening using the same method mentioned above.

\section{Control of Verticillium dahliae by Different Concentrations of Fusarium proliferatum 10R-7}

The biocontrol efficacy of four conidial concentrations of F. proliferatum 10R-7 against $V$. dahliae was evaluated in greenhouse experiments. Seedlings of the cotton variety "JI11" were prepared as described above. Conidia of F. proliferatum $10 \mathrm{R}-7$ were harvested by washing 7 -day-old PDA cultures $\left(25^{\circ} \mathrm{C}\right)$ with autoclaved distilled water, and the resulting mixtures were filtered and the concentration of the conidial suspension was adjusted to $1 \times 10^{7}, 1 \times 10^{6}, 1 \times 10^{5}$, and $1 \times 10^{4}$ conidia/ml. $V$. dahliae V991b was harvested following the method described above, and the concentration was adjusted to $5 \times 10^{6}$ conidia $/ \mathrm{ml}$. The roots of cotton seedlings were submerged in $50 \mathrm{ml}$ of conidial suspension of $V d$ for $20 \mathrm{~min}$ after being submerged in $50 \mathrm{ml}$ of different concentrations of $F$. proliferatum 10R-7 for $10 \mathrm{~min}$. Plants were submerged in $50 \mathrm{ml}$ of the conidial suspension of $\mathrm{Vd}$ for $20 \mathrm{~min}$ after being submerged with $50 \mathrm{ml}$ of sterile water for 10 min were used as controls. Each treatment included 10 plants, and the experiment was replicated three times. The plants were kept at $25 \pm 3^{\circ} \mathrm{C}$ with a 14 -h photoperiod in the greenhouse. The disease severity index was calculated and analyzed according to the method above.

\section{Bioassay of the Volatile Organic Compounds of the Fusarium proliferatum 10R-7}

The F. proliferatum 10R-7 screened in the abovementioned greenhouse experiment was used in this bioassay. The doubledish method was adopted for the bioassay of the volatile organic compounds of the strain 10R-7. The F. proliferatum 10R-7 and $V$. dahliae V991b were incubated at opposite position to form the double dishes. The colony diameter of V991b was measured after 14 days of incubation, and the growth inhibition rate was calculated. The experimental and calculating method followed the descriptions by Zhang et al. (2015). The experiment was repeated twice.

\section{Suppression of Verticillium dahliae by the Culture Filtrates of the Fusarium proliferatum 10R-7}

The F. proliferatum 10R-7 was inoculated in $100 \mathrm{ml}$ of CzapekDox medium in a $250-\mathrm{ml}$ Erlenmeyer flask in shake culture $(150 \mathrm{rpm})$ at $25^{\circ} \mathrm{C}$ for 6 days. The culture filtrates (CFs) were acquired by centrifuging the culture at $6,000 \mathrm{rpm}$ for $10 \mathrm{~min}$ and then filtering through $0.22-\mu \mathrm{m} \mathrm{MS}{ }^{\circledR}$ PES syringe filters. Then, the CFs were added to PDA at a proportion of 1:9 (v/v) with a final CF concentration of $10 \%$. Sterile water was added to PDA as control. Mycelial agar plugs of $V$. dahliae V991b were inoculated on the CF-amended and control PDA. The colony diameter $(C D)$ of $V$. dahliae V991b was measured after growing for 14 days at $25^{\circ} \mathrm{C}$, and the growth inhibition rate was calculated. The percentage $(P)$ of the growth inhibition rate was calculated by the following formula:

$$
P(\%)=\left(C D_{C K}-C D_{C F}\right) / C D_{C K} \times 100
$$

where $C D_{C F}$ represents the colony diameter in the treatment amending with the $C F$ s and $C D_{C K}$ represents the colony diameter in the control treatment. 


\section{Determination of the Antifungal Activity of the Culture Filtrates of Fusarium proliferatum 10R-7}

Two different culture media, potato dextrose broth (PDB) medium and Czapek-Dox medium (CDM), were used to incubate isolate 10R-7 for the production of the antifungal metabolites. The ingredients of the PDB medium were as follows: peeled potato, $200 \mathrm{~g}$; glucose, $20 \mathrm{~g}$; and distilled water, 1,000 ml. The ingredients of Czapek-Dox medium were as follows: $\mathrm{KNO}_{3}$, $2 \mathrm{~g} ; \mathrm{KH}_{2} \mathrm{PO}_{4}, 1 \mathrm{~g} ; \mathrm{MgSO}_{4}, 0.5 \mathrm{~g}$; KCl, $0.5 \mathrm{~g} ; \mathrm{FeSO}_{4}, 0.01 \mathrm{~g}$; sucrose, $30 \mathrm{~g}$; and distilled water, 1,000 ml. The isolate $10 \mathrm{R}-7$ was cultured in a $\mathrm{PDB}$ or $\mathrm{CDM}$ shake culture $(150 \mathrm{rpm})$ at $25^{\circ} \mathrm{C}$ for $3,6,9$, 12,15 , and 18 days. The CFs of isolate 10R-7 from each sampling date were obtained and added to PDA at 2, 4, 6, 8, and 10\% (v/v), and the PDA without any amendment was treated as control. Mycelial agar plugs of $V$. dahliae were inoculated on the PDA and incubated for 14 days at $25^{\circ} \mathrm{C}$. The diameter of each colony was measured and the calculation of the growth inhibition rate was performed as described above.

Moreover, inhibition of spore germination of $V$. dahliae by the CFs of isolate 10R-7 was tested. Plates with water agar (WA) amended with $10 \%$ CFs of isolate 10R-7 were prepared. A $20-\mu 1$ conidial suspension of $V$. dahliae $\left(1 \times 10^{4} \mathrm{conidia} / \mathrm{ml}\right)$ was spread evenly on a Petri dish with a glass rod and cultured at $25^{\circ} \mathrm{C}$. The control treatment consisted of WA amended with sterile water. The cultures were incubated at $25^{\circ} \mathrm{C}$ for $6,12,24$, and $48 \mathrm{~h}$, and at each time point, three dishes for each treatment were sampled and germinated conidia on the media were observed, with 100 conidia per dish. Moreover, 20 germinated conidia on each medium were randomly selected, and the length of the germ tubes was measured.

\section{Changes in Biomass and $\mathrm{pH}$ in the Culture Filtrates of Fusarium proliferatum 10R-7}

A mycelial agar plug of $F$. proliferatum 10R-7 was shakeinoculated $\left(150 \mathrm{rpm}, 25^{\circ} \mathrm{C}\right)$ in CDM for $2,4,6,8$, and 10 days. At each time point, three cultures were sampled, and the mycelial mass in each culture was collected by filtering, dried at $80^{\circ} \mathrm{C}$ for $6 \mathrm{~h}$, and weighed. The filtrates were centrifuged at $6,000 \mathrm{rpm}$ at $4^{\circ} \mathrm{C}$, and the $\mathrm{pH}$ value was measured using a $\mathrm{pH}$ meter. The concentration of fusaric acid in the culture filtrates was measured in a spectrophotometer (BioTek, Epoch, Winooski, VT, United States) at $273 \mathrm{~nm}$ using pure fusaric acid (Shanghai TCI Development Co., Ltd., Shanghai, China) as reference.

\section{Identification of the Antifungal Substances Produced by Fusarium proliferatum 10R-7}

Macroporous resin granules (5 g) were added to $100 \mathrm{ml}$ of culture filtrates (PDB or CDM) of isolate 10R-7, and the mixture was consistently shaken $(150 \mathrm{rpm})$ at $25^{\circ} \mathrm{C}$ for $10 \mathrm{~h}$. The resin was then washed three times with sterile water and the precipitate was resuspended with $10 \mathrm{ml}$ methanol. The mixture was rotarily evaporated and redissolved in $1 \mathrm{ml}$ of anhydrous ethanol. The solution was loaded in a high-performance liquid chromatography (HPLC) column for semipreparation. The fractions that exhibited antifungal activity against $V$. dahliae were subjected to electrospray ionization mass spectrometric (ESI-MS) analysis (Lyu et al., 2017).

\section{Antifungal Activity of Fusaric Acid Against Verticillium dahliae}

Pure fusaric acid (purity: 98\%) was purchased from Shanghai TCI Development Co., Ltd. First, $0.01 \mathrm{~g}$ of fusaric acid was dissolved in $1 \mathrm{ml}$ of anhydrous ethanol to prepare the mother solution at $10 \mathrm{mg} / \mathrm{ml}$; then, the mother solution was added to PDA to final concentrations of $5,10,20,25,35,40,60$, and $80 \mu \mathrm{g} / \mathrm{ml}$. PDA without amendment of fusaric acid was used as control. There were three replicates for each treatment. V. dahliae V991b was inoculated on PDA for different treatments and incubated at $25^{\circ} \mathrm{C}$ for 7 days. The colony diameter in each dish was measured, and the inhibition rate of fusaric acid on the growth of $V$. dahliae was calculated using the formula mentioned above. The experiment was repeated twice.

\section{Influence of Fusaric Acid on the Germination of Cotton Seeds and Growth of Cotton Seedlings}

The mother solution of $10 \mathrm{mg} / \mathrm{ml}$ as mentioned above was diluted with sterile water to prepare solutions of different concentrations of fusaric acid $(5,10$, and $20 \mu \mathrm{g} / \mathrm{ml})$. Cotton seeds (JI11) were surface-sterilized with sodium hypochlorite for $2 \mathrm{~min}$, rinsed with sterile water several times, and then soaked for $30 \mathrm{~min}$ in solutions of fusaric acid at different concentrations. Cotton seeds soaked in sterile water for 30 min were used as controls. Then, the seeds were sown in nutrient vermiculite in plastic pots $(10 \mathrm{~cm} \times 9 \mathrm{~cm}$, diameter $\times$ height $)$ with four seeds in each pot $(20$ pots for each treatment). The pots were placed in a greenhouse $\left(25 \pm 3^{\circ} \mathrm{C}\right)$ and watered as required. The number of germinated cotton seeds in each pot was counted after 5 days, and the germination rates were calculated. Then, the seedlings in the pots were thinned to have one seedling per pot. At 20 days postsowing, 20 cotton plants were carefully uprooted and washed under tap water. Growth indices such as stem height, root length, fresh weight, and dry weight $\left(60^{\circ} \mathrm{C}, 48 \mathrm{~h}\right)$ were individually measured. This experiment was performed twice.

\section{Antifungal Activity of Fusaric Acid on Cotton Verticillium Wilt in Greenhouse Experiments}

Cotton seedlings were grown in pots to the two-true-leaf stage, and a conidial suspension of $V$. dahliae V991b $\left(5 \times 10^{6}\right.$ conidia $\left./ \mathrm{ml}\right)$ was prepared. The mother solution of fusaric acid was added to a conidial suspension of $V$. dahliae V991b to make the solutions of different concentrations from 2.5 to $80 \mu \mathrm{g} / \mathrm{ml}$. The experiment was carried out in three trials. Trial 1 included four concentrations of fusaric acid, namely, 2.5, 5,15 , and $25 \mu \mathrm{g} / \mathrm{ml}$. Trial 2 included three concentrations of fusaric acid, namely, 10, 20, and $40 \mu \mathrm{g} / \mathrm{ml}$. Trial 3 included three concentrations of fusaric acid, namely, 20, 40, and $80 \mu \mathrm{g} / \mathrm{ml}$. 
Then, the roots of cotton seedlings (10 per treatment, three replicates per treatment) were drenched in a conidial suspension of $V$. dahliae V991b $\left(5 \times 10^{6}\right.$ conidia/ml $)$ mixed with fusaric acid for $20 \mathrm{~min}$. Seedlings inoculated with the $V$. dahliae only and sterile water only served as positive and negative controls, respectively. The seedlings were transplanted into nutrition vermiculite in plastic pots $(12 \mathrm{~cm} \times 10 \mathrm{~cm}$, diameter $\times$ height $)$, with three to four seedlings per pot and three pots per treatment after inoculation. The temperature was maintained at $25 \pm 3^{\circ} \mathrm{C}$, and the seedlings were watered to maintain an appropriate humidity. The survey method was the same as those mentioned above. The experiment was repeated twice.

\section{Colonization of Fusarium proliferatum 10R-7 on Cotton Roots}

The GFP-labeled strain 10R-7GFP was constructed according to Agrobacterium tumefaciens-mediated transformation (Zhang et al., 2015). The plasmid camp-GFP containing the GFP gene was provided by Y. J. Huang (School of Life and Medical Sciences, University of Hertfordshire, AL109, Hatfield, AB, United Kingdom).

Isolate 10R-7GFP was derived from the parental isolate 10R-7 and contained a green fluorescent protein gene. Both isolates had similar growth rates on PDA at $25^{\circ} \mathrm{C}$ (Supplementary Table 2), and isolate $10 \mathrm{R}-7 \mathrm{GFP}$ was stored at $-80^{\circ} \mathrm{C}$ in $20 \%$ glycerol (v/v). Pure cultures of F. proliferatum 10R-7 and 10R-7GFP were also compared under a microscope (Olympus IX81) equipped with an Olympus U-RFL-T.

To examine the colonization of $F$. proliferatum 10R-7GFP in cotton seedlings, the roots of the two-true-leaf stage cotton seedlings were submerged in a spore suspension of the isolate 10R-7GFP $\left(5 \times 10^{6}\right.$ conidia/ml $)$ for $20 \mathrm{~min}$, and the wildtype isolate 10R-7 and sterile water were used as controls. The seedlings were individually transplanted into nutrition vermiculite in plastic pots $(12 \mathrm{~cm} \times 10 \mathrm{~cm}$, diameter $\times$ height at three seedlings per pot, and the temperature was maintained at $25 \pm 3^{\circ} \mathrm{C}$. Then, the seedlings were sampled at $0,1,2$, and 7 days postinoculation. The taproots of the samples were cut into slices transversely and longitudinally using a surgical blade. Then, the thin slices were put on the glass slide and observed under a microscope (Olympus IX81) equipped with an Olympus U-RFLT. The MShot Image Analysis System was used for image analysis.

\section{Data Analysis}

The Statistical Analysis Software (IBM SPSS Statistics 22.0) was used to analyze the data acquired. Significant differences between different treatments were determined using Duncan's test $(P<0.05)$ via one-way ANOVA.

\section{RESULTS}

\section{Diversity of the Endophytic Fungi in Cotton}

One hundred and eighty cotton samples were collected in the test field and 93 isolates of endophytic fungi were isolated from the samples. Twenty species were identified among these isolates according to morphological characteristics and molecular identification (Table 1). The isolated endophytic fungi in cotton were diverse, with Shannon's diversity $\left(H^{\prime}\right)$ and Simpson's diversity $(D)$ indices reaching up to 3.848 and 0.915 , respectively. Fusarium spp. and Alternaria spp. each accounted for 18 of the 93 isolates (19.4\% of the total each), and they were the two most dominant genera. Phomopsis spp. and Plectosphaerella cucumerina each accounted for 10 of 93 isolates. Eight of 93 isolates belonged to Acrocalymma vagum, seven isolates to Diaporthe spp., five isolates belonged to Colletotrichum gloeosporioides, five isolates belonged to Botryosphaeria dothidea, three isolates to Clonostachys rosea, two isolates belonged to Leptosphaerulina chartarum, and two isolates belonged to Trichoderma spp. The remaining five fungal species belonged to Albifimbria viridis, Macrophomina phaseolina, Phaeosphaeria spp., Phoma spp., and $V$. dahliae. The diversity of endophytic fungi in the roots and stems was different. Among the 93 fungal isolates, 23 were found in the roots and 70 were from the stems. The values for Shannon's diversity and Simpson's diversity indices were 2.523 and 0.802 in the roots and 3.593 and 0.895 in the stems, respectively (Table 2). Four species, namely, A. vagum, F. proliferatum, Phomopsis spp., and P. cucumerina, were simultaneously isolated from the roots and stems of cotton. Sixteen fungal species were isolated from one of the two cotton tissues.

\section{Biocontrol Efficacy in Greenhouse Experiments}

Seventy-two spore-producing isolates were tested for the control of Verticillium wilt of cotton in the greenhouse. The results are shown in Supplementary Table 1. Five isolates (10R-2, 10R7, 10R-9, 6R-1, and 17R-8) were obtained through multiple rounds of screening, and the average control efficacy against Verticillium wilt was higher than $50 \%$ (Table 3). The disease severity and pathogenic incidence were all significantly $(P<0.05)$ reduced relative to those of the pathogen-only control. In trial 1 , the biocontrol efficacy of the five isolates 10R-2, 10R-7, 10R9, 6R-1, and 17R-8 against cotton Verticillium wilt ranged from $58.1 \%$ to $88.9 \%$. In trial 2 , the biocontrol efficacy against cotton Verticillium wilt ranged from $44.9 \%$ to $80.2 \%$. In trial 3 , the biocontrol efficacy against cotton Verticillium wilt ranged from 34.4 to $63.4 \%$. The results of the three trials showed that the average biocontrol efficacy of 10R-2, 10R-7, 10R-9, 6R-1, and 17R-8 against cotton Verticillium wilt were 59.0, 70.0, 51.8, 58.0, and $65.1 \%$, respectively, and that isolate $10 \mathrm{R}-7$ showed the best inhibitory ability (Figure 1). Among them, isolates 10R-2, 10R7, and 10R-9 belonged to F. proliferatum, and isolates 6R-1 and 17R-8 belonged to $F$. solani.

\section{Suppression Efficacy of the Volatiles and Culture Filtrates of Fusarium proliferatum 10R-7}

The F. proliferatum isolate 10R-7 could produce antifungal substances inhibitory to $V$. dahliae. The inhibition percentages of the volatiles of the isolate $10 \mathrm{R}-7$ were $69.70 \%$. The culture filtrates 
TABLE 1 | Identification of the endophytic fungi from cotton by analysis of the ITS (ITS1-5.8S rDNA-ITS2) sequences.

\begin{tabular}{|c|c|c|c|}
\hline Isolate & Identity to the closest species (GenBank acc. no.) & Isolate & Identity to the closest species (GenBank acc. no.) \\
\hline $5 R-7$ & $100 \%$ to Acrocalymma vagum (KF494165) & $3 S-5$ & 99\% to Fusarium oxysporum (MG136706) \\
\hline $5 R-9$ & 99\% to Acrocalymma vagum (KP784427) & $7 S-3$ & 99\% to Fusarium oxysporum (KF264963) \\
\hline $5 S-4$ & 99\% to Acrocalymma vagum (KP784427) & $17 S-3$ & $100 \%$ to Fusarium oxysporum (KU527803) \\
\hline 10R-3 & 99\% to Acrocalymma vagum (KX064982) & $1 S-2$ & 99\% to Fusarium proliferatum (MF510820) \\
\hline 10R-4 & 99\% to Acrocalymma vagum (KX064982) & $1 S-7$ & $100 \%$ to Fusarium proliferatum (MF614933) \\
\hline $17 R-12$ & 99\% to Acrocalymma vagum (KX064982) & $2 S-5$ & 99\% to Fusarium proliferatum (MF510820) \\
\hline $17 \mathrm{R}-13$ & 99\% to Acrocalymma vagum (KX064982) & $3 S-4$ & 99\% to Fusarium proliferatum (GQ924905) \\
\hline 17R-14 & 99\% to Acrocalymma vagum (KX064987) & 10R-2 & 99\% to Fusarium proliferatum (MG543724) \\
\hline $6 S-6$ & 99\% to Albifimbria viridis (KU845899) & $10 R-5$ & 99\% to Fusarium proliferatum (MF614934) \\
\hline $1 S-4$ & 99\% to Alternaria alternata (KX783388) & 10R-7 & 99\% to Fusarium proliferatum (MG543724) \\
\hline $1 S-8$ & $100 \%$ to Alternaria alternata (KJ526175) & $10 R-9$ & 99\% to Fusarium proliferatum (MG543770) \\
\hline $2 S-3$ & 99\% to Alternaria alternata (KR912224) & $10 S-7$ & 99\% to Fusarium proliferatum (FJ648201) \\
\hline $2 S-4$ & 99\% to Alternaria alternata (KX783403) & $6 \mathrm{R}-1$ & 99\% to Fusarium solani (KX064991) \\
\hline $3 S-6$ & 99\% to Alternaria alternata (KP638335) & $7 \mathrm{R}-2$ & 99\% to Fusarium solani (KX064991) \\
\hline $3 S-7$ & 99\% to Alternaria alternata (KX858844) & $7 R-3$ & 99\% to Fusarium solani (MF800959) \\
\hline $3 S-8$ & 99\% to Alternaria alternata (KY114869) & $10 R-6$ & 99\% to Fusarium solani (KU528855) \\
\hline $4 S-8$ & 99\% to Alternaria alternata (KF380821) & 17R-8 & 99\% to Fusarium solani (MF800959) \\
\hline $4 S-18$ & 99\% to Alternaria alternata (KU982599) & $5 S-5$ & 99\% to Fusarium nematophilum (KU324800) \\
\hline $4 S-21$ & 99\% to Alternaria alternata (KU533841) & $4 S-20$ & 99\% to Leptosphaerulina chartarum (KJ584553) \\
\hline $4 S-22$ & 99\% to Alternaria alternata (KX783388) & $4 S-27$ & 99\% to Leptosphaerulina chartarum (KJ584553) \\
\hline $4 S-39$ & 99\% to Alternaria alternata (KJ526174) & $17 \mathrm{R}-15$ & 99\% to Macrophomina phaseolina (FJ395243) \\
\hline $4 S-40$ & 100\% to Alternaria alternata (KX783405) & $4 S-24$ & 99\% to Phaeosphaeria sp. (HQ914835) \\
\hline $6 S-1$ & 99\% to Alternaria alternata (KF380814) & $4 S-36$ & 99\% to Phoma sp. (KT355015) \\
\hline $17 S-4$ & 99\% to Alternaria alternata (KF380821) & $1 S-6$ & 99\% to Phomopsis sp. (MF800891) \\
\hline $1 S-3$ & $100 \%$ to Alternaria tenuissima (KU324783) & $5 S-2$ & 99\% to Phomopsis sp. (KC172081) \\
\hline $4 S-38$ & 99\% to Alternaria tenuissima (KC337038) & $6 S-4$ & 99\% to Phomopsis sp. (KX722227) \\
\hline $10 S-1$ & 99\% to Alternaria tenuissima (KX065003) & $7 \mathrm{R}-4$ & 99\% to Phomopsis sp. (KC172081) \\
\hline $1 S-10$ & 99\% to Botryosphaeria dothidea (KF293751) & $7 S-2$ & 99\% to Phomopsis sp. (KC172081) \\
\hline $5 S-7$ & 100\% to Botryosphaeria dothidea (KR709076) & $7 S-4$ & 99\% to Phomopsis sp. (KC172081) \\
\hline $5 S-8$ & 100\% to Botryosphaeria dothidea (KF293759) & $7 S-5$ & 99\% to Phomopsis sp. (KC172081) \\
\hline $6 S-5$ & 99\% to Botryosphaeria dothidea (KM433848) & $7 S-6$ & 99\% to Phomopsis sp. (KC172081) \\
\hline $9 S-1$ & 99\% to Botryosphaeria dothidea (KF293867) & $8 S-3$ & 99\% to Phomopsis sp. (KC172081) \\
\hline $1 S-16$ & 99\% to Clonostachys rosea (MF567519) & $8 S-4$ & 99\% to Phomopsis sp. (KC172081) \\
\hline $9 S-2$ & $100 \%$ to Clonostachys rosea (MF663694) & $1 \mathrm{R}-1$ & 99\% to Plectosphaerella cucumerina (HQ914913) \\
\hline $9 S-3$ & 99\% to Clonostachys rosea (KX783336) & $1 S-15$ & 99\% to Plectosphaerella cucumerina (HQ914913) \\
\hline $1 S-9$ & 99\% to Colletotrichum gloeosporioides (KM520010) & $4 S-14$ & 99\% to Plectosphaerella cucumerina (HQ914913) \\
\hline $4 S-7$ & 99\% to Colletotrichum gloeosporioides (MF800897) & $4 S-17$ & 99\% to Plectosphaerella cucumerina (HQ914913) \\
\hline $4 S-9$ & 99\% to Colletotrichum gloeosporioides (JN887346) & $4 S-23$ & 99\% to Plectosphaerella cucumerina (HQ914913) \\
\hline $10 S-4$ & 99\% to Colletotrichum gloeosporioides (JN887348) & $5 S-3$ & 99\% to Plectosphaerella cucumerina (HQ914913) \\
\hline $10 S-14$ & 99\% to Colletotrichum gloeosporioides (MF800894) & 10R-1 & 99\% to Plectosphaerella cucumerina (KC756235) \\
\hline $6 S-2$ & 99\% to Diaporthe sp. (KF928282) & $10 \mathrm{R}-8$ & 99\% to Plectosphaerella cucumerina (HQ914913) \\
\hline $6 S-3$ & 99\% to Diaporthe sp. (KR709022) & $10 S-8$ & 99\% to Plectosphaerella cucumerina (HQ914913) \\
\hline $7 \mathrm{~S}-7$ & 99\% to Diaporthe sp. (KC172081) & $10 S-18$ & 99\% to Plectosphaerella cucumerina (HQ914913) \\
\hline $9 S-14$ & 99\% to Diaporthe sp. (KU324789) & $7 R-5$ & 99\% to Trichoderma sp. (KR868247) \\
\hline $17 S-1$ & 100\% to Diaporthe sp. (MF686806) & $7 \mathrm{R}-6$ & 99\% to Trichoderma sp. (KP689168) \\
\hline $17 S-2$ & 99\% to Diaporthe sp. (MF686806) & $5 S-10$ & 99\% to Verticillium dahliae (KY454838) \\
\hline 17S-6 & 99\% to Diaporthe sp. (KC172081) & & \\
\hline
\end{tabular}

of isolate 10R-7 were obtained by cultivation of the isolate for 7 days in CDM. The culture filtrates were amended into PDA at the volume ratio of $10 \%$ to suppress the growth of $V$. dahliae. The results showed that the inhibition percentage reached up to $100 \%$ for the culture filtrates of isolate 10R-7 (Table 4).

\section{Control Efficacy of Fusarium proliferatum 10R-7 at Different Dosages}

The ability of the isolate 10R-7 at four different concentrations to control $V$. dahliae was evaluated in a greenhouse. When the soil was inoculated with a conidial suspension of $F$. proliferatum 
TABLE 2 | Endophytic fungi in cotton tissue in Wuhan of central China.

\begin{tabular}{|c|c|c|c|c|}
\hline \multirow[t]{2}{*}{ Species } & \multicolumn{3}{|c|}{ Number of fungal isolates } & \multirow{2}{*}{$\begin{array}{c}\text { Frequency } \\
(\%)\end{array}$} \\
\hline & Root & Stem & Total & \\
\hline Acrocalymma vagum & 7 & 1 & 8 & 8.6 \\
\hline Albifimbria viridis & $\mathrm{NI}$ & 1 & 1 & 1.1 \\
\hline Alternaria alternata & $\mathrm{NI}$ & 15 & 15 & 16.1 \\
\hline Alternaria tenuissima & $\mathrm{NI}$ & 3 & 3 & 3.2 \\
\hline Botryosphaeria dothidea & $\mathrm{NI}$ & 5 & 5 & 5.4 \\
\hline Clonostachys rosea & $\mathrm{NI}$ & 3 & 3 & 3.2 \\
\hline Colletotrichum gloeosporioides & $\mathrm{NI}$ & 5 & 5 & 5.4 \\
\hline Diaporthe sp. & $\mathrm{NI}$ & 7 & 7 & 7.5 \\
\hline Fusarium oxysporum & $\mathrm{NI}$ & 3 & 3 & 3.2 \\
\hline Fusarium proliferatum & 4 & 5 & 9 & 9.7 \\
\hline Fusarium solani & 5 & $\mathrm{NI}$ & 5 & 5.4 \\
\hline Fusarium nematophilum & $\mathrm{NI}$ & 1 & 1 & 1.1 \\
\hline Leptosphaerulina chartarum & $\mathrm{NI}$ & 2 & 2 & 2.2 \\
\hline Macrophomina phaseolina & 1 & $\mathrm{NI}$ & 1 & 1.1 \\
\hline Phaeosphaeria sp. & $\mathrm{NI}$ & 1 & 1 & 1.1 \\
\hline Phoma sp. & $\mathrm{NI}$ & 1 & 1 & 1.1 \\
\hline Phomopsis sp. & 1 & 9 & 10 & 10.8 \\
\hline Plectosphaerella cucumerina & 3 & 7 & 10 & 10.8 \\
\hline Trichoderma sp. & 2 & $\mathrm{NI}$ & 2 & 2.2 \\
\hline Verticillium dahliae & $\mathrm{NI}$ & 1 & 1 & 1.1 \\
\hline Total number of isolates $(N)$ & 23 & 70 & 93 & - \\
\hline Species richness $(S)$ & 7 & 18 & 20 & - \\
\hline Shannon index of diversity $\left(H^{\prime}\right)$ & 2.523 & 3.593 & 3.848 & - \\
\hline Simpson's diversity index $(D)$ & 0.802 & 0.895 & 0.915 & - \\
\hline
\end{tabular}

NI, not isolated.

10R-7 at $1 \times 10^{4}, 1 \times 10^{5}, 1 \times 10^{6}$, or $1 \times 10^{7}$ conidia/ml before inoculation with a spore suspension of $V$. dahliae, a significant difference $(P<0.05)$ in disease index was observed between the treatments of isolate 10R-7 $\left(1 \times 10^{6}\right.$ and $1 \times 10^{7}$ conidia/ml $)$ and the control. The biocontrol efficacy increased with increasing spore concentration for isolate 10R-7. The biocontrol efficacy values for these spore concentrations were 8.7, 16.2, 40.7, and $70.8 \%$, respectively, at $20 \mathrm{dpi}$, and $-3.5,7.9,21.1$, and $54.4 \%$, respectively, at $25 \mathrm{dpi}$ (Table 5). The efficacy values at 20 and 25 dpi were positively correlated with the spore concentration with correlation coefficients of 0.971 and 0.963 , respectively.

\section{Suppression of Verticillium dahliae by the Culture Filtrates of Fusarium proliferatum 10R-7}

The ability of F. proliferatum 10R-7 culture filtrates in PDB and $\mathrm{CDM}$ to suppress $V$. dahliae was evaluated. The culture filtrates showed different inhibitory activities. The inhibition percentage by the culture filtrates in CDM was significantly higher $(P<0.05)$ than that of the culture filtrates in PDB. The inhibition percentage was $100 \%$ for the $10 \%$ 6-day-old CDM culture filtrates and $8.9 \%$ for the 6-day-old PDB culture filtrates (Figure 2). The inhibition percentage was $100 \%$ for the CDM culture filtrates even at the concentration of $4 \%$ (Figure 3 ).
TABLE 3 | Control effects of the five antagonists of controlling cotton Verticillium wilt in greenhouse.

\begin{tabular}{|c|c|c|}
\hline Treatment & Disease index & Biocontrol efficacy (\%) \\
\hline \multicolumn{3}{|l|}{ Trial 1} \\
\hline F. proliferatum 10R-2 + V. dahliae & $34.3 \pm 2.2 b^{1}$ & $58.1 d^{1}$ \\
\hline F. proliferatum 10R-7 + V. dahliae & $9.1 \pm 1.5 \mathrm{~d}$ & $88.9 \mathrm{a}$ \\
\hline F. proliferatum 10R-9 + V. dahliae & $19.6 \pm 6.0 \mathrm{c}$ & $76.0 \mathrm{c}$ \\
\hline F. solani $6 \mathrm{R}-1+$ V. dahliae & $14.3 \pm 2.4 \mathrm{~cd}$ & $82.5 \mathrm{~b}$ \\
\hline F. solani 17R-8 + V. dahliae & $16.1 \pm 3.6 \mathrm{c}$ & $80.4 \mathrm{~b}$ \\
\hline V. dahliae alone & $81.8 \pm 1.5 \mathrm{a}$ & 0.0 e \\
\hline Control (water) & 0.0 & - \\
\hline \multicolumn{3}{|l|}{ Trial 2} \\
\hline F. proliferatum 10R-2 + V. dahliae & $17.3 \pm 2.6 d^{1}$ & $80.2 a^{1}$ \\
\hline F. proliferatum 10R-7 + V. dahliae & $29.2 \pm 2.8 c$ & $66.7 \mathrm{~b}$ \\
\hline F. proliferatum 10R-9 + V. dahliae & $48.2 \pm 6.0 \mathrm{~b}$ & $44.9 \mathrm{~d}$ \\
\hline F. solani $6 \mathrm{R}-1+$ V. dahliae & $39.6 \pm 8.3 b$ & $54.8 \mathrm{c}$ \\
\hline F. solani 17R-8 + V. dahliae & $42.4 \pm 3.2 b$ & $51.6 \mathrm{c}$ \\
\hline V. dahliae alone & $87.5 \pm 2.8 \mathrm{a}$ & 0.0 e \\
\hline Control (water) & 0.0 & - \\
\hline \multicolumn{3}{|l|}{ Trial 3} \\
\hline F. proliferatum 10R-2 + V. dahliae & $45.0 \pm 1.1 b^{1}$ & $38.6 c^{1}$ \\
\hline F. proliferatum 10R-7 + V. dahliae & $33.3 \pm 6.8 c$ & $54.5 \mathrm{~b}$ \\
\hline F. proliferatum 10R-9 + V. dahliae & $48.1 \pm 3.9 b$ & $34.4 \mathrm{c}$ \\
\hline F. solani $6 \mathrm{R}-1+$ V. dahliae & $46.4 \pm 4.8 b$ & $36.6 \mathrm{c}$ \\
\hline F. solani 17R-8 + V. dahliae & $26.8 \pm 2.4 c$ & $63.4 \mathrm{a}$ \\
\hline V. dahliae alone & $73.3 \pm 4.4 \mathrm{a}$ & $0.0 \mathrm{~d}$ \\
\hline Control (water) & 0.0 & - \\
\hline
\end{tabular}

${ }^{1}$ Means $\pm S E$ within each column in each trial followed by the same letters are not significantly different $(P>0.05)$ according to Duncan's multiple test.

In the conidial germination test $\left(\mathrm{WA}, 25^{\circ} \mathrm{C}\right.$ ), the average spore germination rate of $V$. dahliae was $51.7 \%$ at $6 \mathrm{~h}$ postincubation (hpi), $90.7 \%$ at $12 \mathrm{hpi}$, and $100 \%$ at 24 and $48 \mathrm{hpi}$ in the control treatment without amendment of the cultures of 10R-7 in WA (Figure 4). The average length of the germ tubes was $31.5 \mu \mathrm{m}$ at $12 \mathrm{hpi}$ and $294.3 \mu \mathrm{m}$ at $48 \mathrm{hpi}$. When the culture filtrates of 10R-7 were amended in WA (10\%), however, the percentages of germinated conidia were reduced to $7.7,15.0$, and $49.3 \%$ at 6,12 , and $24 \mathrm{hpi}$, respectively, and at $48 \mathrm{hpi}$, the value reached up to $100.0 \%$. The average length of the germ tubes was reduced to 13.0 , 23.8 , and $49.8 \mu \mathrm{m}$, at 12,24 , and $48 \mathrm{hpi}$, respectively (Figure 4 ). The results indicated that the culture filtrates of isolate 10R-7 had inhibitory effects both on spore germination and on elongation of germ tubes of $V$. dahliae.

\section{Time Course of Biomass, $\mathrm{pH}$, and Content of Fusaric Acid in the Cultures of Fusarium proliferatum 10R-7}

The biomass of $F$. proliferatum 10R-7 in CDM cultures $\left(25^{\circ} \mathrm{C}\right)$ increased with time, reached the maximum value at $8 \mathrm{dpi}$, and had a small decrease at $10 \mathrm{dpi}$. The biomass rapidly increased threefold from 2 to $4 \mathrm{dpi}$. The $\mathrm{pH}$ value increased from 7.3 to 8.4 from 2 to $10 \mathrm{dpi}$.

The inhibition percentages against $V$. dahliae reached $100 \%$ at $4 \mathrm{dpi}$. At the same time, the absorption values of the culture 

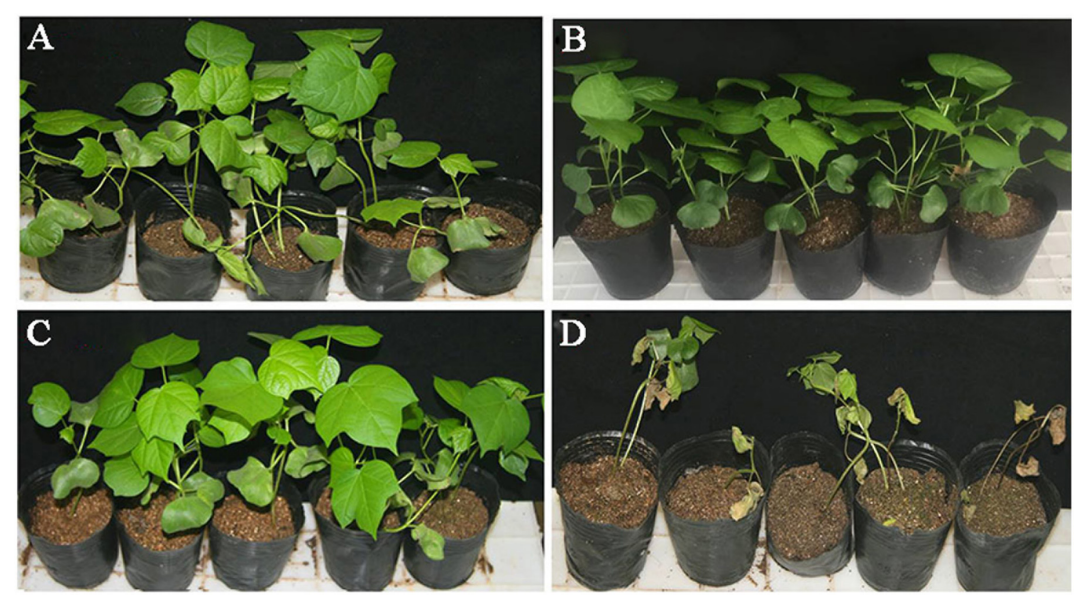

FIGURE 1 | Efficacy of $F$. proliferatum 10R-7 in suppression of Verticillium wilt of cotton caused by Verticillium dahliae ( $25 \pm 3^{\circ} \mathrm{C}, 30$ days postinoculation) in a greenhouse experiment. (A) Cotton seedlings treated with sterile water control (CK). (B) Cotton seedlings treated with F. proliferatum $10 \mathrm{R}-7$ alone (10R-7 alone). (C) Cotton seedlings treated with F. proliferatum 10R-7 plus V. dahliae (10R-7 + Vd). (D) Cotton seedlings treated with V. dahliae alone (Vd alone).

TABLE 4 | Inhibition of mycelial growth of Verticillium dahliae by the culture filtrates and the volatiles produced by the F. proliferatum $10 R-7$.

\begin{tabular}{|c|c|c|c|c|}
\hline \multirow[t]{2}{*}{ Isolate } & \multicolumn{2}{|c|}{ Culture filtrates } & \multicolumn{2}{|c|}{ Volatiles } \\
\hline & Colony diameter (cm) & Inhibition percentage & Colony diameter (cm) & Inhibition percentage \\
\hline F. proliferatum 10R-7 & $0.0 \pm 0.0 b^{1}$ & $100.0 \%$ a & $1.9 \pm 0.4 b^{1}$ & $69.7 \%$ a \\
\hline Control & $5.9 \pm 0.1 \mathrm{a}$ & - & $6.3 \pm 0.4 \mathrm{a}$ & - \\
\hline
\end{tabular}

${ }^{1}$ Means \pm SE within each column in each trial followed by the same letters are not significantly different $(P>0.05)$ according to Duncan's multiple test.

TABLE 5 | Effect of different conidial concentrations of $F$. proliferatum 10R-7 on suppression of cotton Verticillium wilt caused by $V$. dahliae in greenhouse.

\begin{tabular}{|c|c|c|c|c|c|}
\hline \multicolumn{2}{|c|}{ Treatment } & \multicolumn{2}{|c|}{20 days postinoculation } & \multicolumn{2}{|c|}{25 days postinoculation } \\
\hline F. proliferatum & V. dahliae & Disease index & Biocontrol efficacy (\%) & Disease index & Biocontrol efficacy (\%) \\
\hline $1 \times 10^{4}$ conidia $/ \mathrm{ml}$ & $5 \times 10^{6}$ conidia $/ \mathrm{ml}$ & $80.8 \pm 5.2 a^{1}$ & 8.7 & $98.3 \pm 2.9 \mathrm{a}^{1}$ & -3.5 \\
\hline $1 \times 10^{5}$ conidia $/ \mathrm{ml}$ & $5 \times 10^{6}$ conidia $/ \mathrm{ml}$ & $74.2 \pm 5.2 \mathrm{a}$ & 16.2 & $87.5 \pm 5.0 \mathrm{ab}$ & 7.9 \\
\hline $1 \times 10^{6}$ conidia $/ \mathrm{ml}$ & $5 \times 10^{6}$ conidia $/ \mathrm{ml}$ & $52.5 \pm 10.0 b$ & 40.7 & $75.0 \pm 15.2 b$ & 21.1 \\
\hline $1 \times 10^{7}$ conidia $/ \mathrm{ml}$ & $5 \times 10^{6}$ conidia $/ \mathrm{ml}$ & $25.8 \pm 9.5 c$ & 70.8 & $43.3 \pm 15.1 \mathrm{c}$ & 54.4 \\
\hline 0 (water) & $5 \times 10^{6}$ conidia $/ \mathrm{ml}$ & $88.5 \pm 9.6 \mathrm{a}$ & - & $95.0 \pm 8.7 \mathrm{ab}$ & - \\
\hline
\end{tabular}

${ }^{1}$ Means \pm SE within each column in each trial followed by the same letters are not significantly different $(P>0.05)$ according to Duncan's multiple test.

filtrates at $273 \mathrm{~nm}$ at different cultivation times were measured to calculate the fusaric acid content. The fusaric acid content increased rapidly when the cultivation time increased from 2 to $4 \mathrm{dpi}$ and increased slowly when the cultivation time further increased from 4 to $10 \mathrm{dpi}$ (Figure 5). These results suggest that the fusaric acid content change was consistent with the change in the inhibition activity of the culture filtrates against $V$. dahliae.

\section{Identification of the Antifungal Substances Produced by Fusarium proliferatum 10R-7}

High-performance liquid chromatography and ESI-MS analyses were applied to analyze the antifungal substances in the culture filtrates of $F$. proliferatum 10R-7. ESI-MS $(3.5 \mathrm{kV})$ showed a molecular ion peak at $m / z 180.02(\mathrm{M}+\mathrm{H})^{+}$and $178.2(\mathrm{M}-\mathrm{H})^{-}$, and the ESI-MS molecular weight was 179.2 Da. Based on these data, the molecular formula of the antifungal substance was inferred to be $\mathrm{C}_{10} \mathrm{H}_{13} \mathrm{NO}_{2}$. The $\mathrm{UV}$ maximum absorbance was at $270.9 \mathrm{~nm}$. We detected fusaric acid in the culture filtrates of F. proliferatum 10R-7 using HPLC and ESI-MS analysis. The HPLC chromatography, TIC, and the UV absorption results of fusaric acid were similar with those of the culture filtrates (Supplementary Figure 1). It was therefore inferred that the antifungal substance in the culture filtrates is fusaric acid. However, the culture filtrates in CDM gave UV maximum absorbance at $272.8 \mathrm{~nm}$, while the culture filtrates in PDB gave UV maximum absorbance at $309.8 \mathrm{~nm}$ (Supplementary Figure 2). This result showed that the secondary metabolites of F. proliferatum 10R-7 in CDM and PDB differed. 


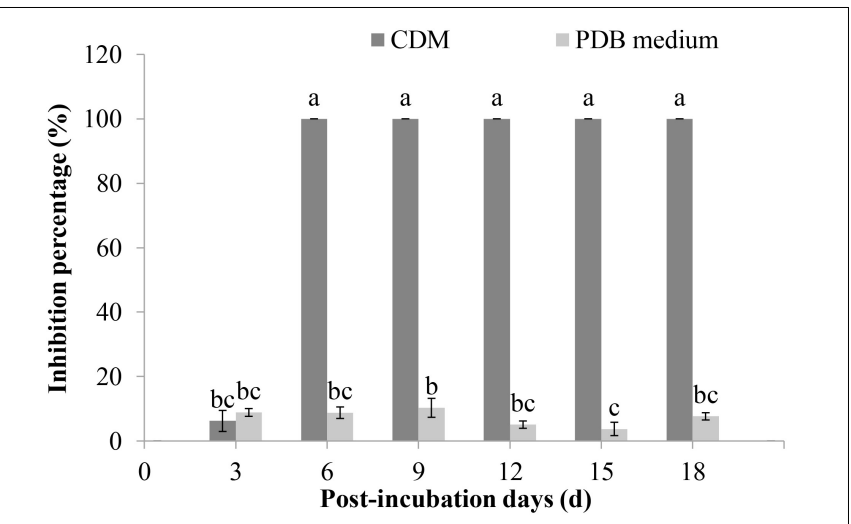

FIGURE 2 | Inhibition rates of $V$. dahliae by the culture filtrates of F. proliferatum 10R-7 in CDM (Czapek-Dox medium) and PDB medium at different culture times.

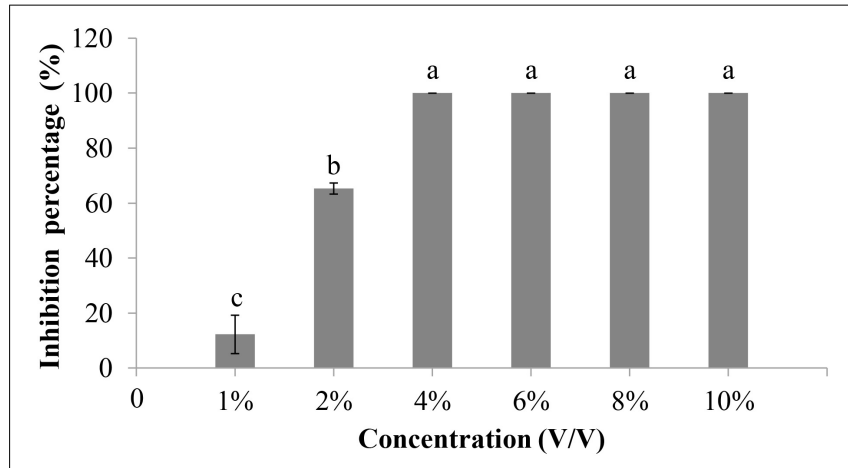

FIGURE 3 | Inhibitory rates of culture filtrates of $F$. proliferatum 10R-7 of different concentrations on $V$. dahliae.

\section{Antifungal Activity of Fusaric Acid}

Fusaric acid solution suppressed the growth of $V$. dahliae. The inhibition percentages at 5 and $10 \mu \mathrm{g} / \mathrm{ml}$ were 40.3 and $57.9 \%$, respectively, and the inhibition percentage reached $100 \%$ when the concentration was $20 \mu \mathrm{g} / \mathrm{ml}$ or higher (Figure 6). These results also verified that fusaric acid may be the antifungal substance in the culture filtrates of F. proliferatum 10R-7.

\section{Influence of Fusaric Acid Concentrations on the Growth of Cotton Seedlings}

High concentrations of fusaric acid had a negative effect on the growth of cotton seedlings. When the concentration was reduced, the negative effect decreased. We surveyed the growth index of the cotton seedlings after treatments with different concentrations $(5,10$, and $20 \mu \mathrm{g} / \mathrm{ml})$. The results showed that fusaric acid at $5 \mu \mathrm{g} / \mathrm{ml}$ did not produce a negative effect on the growth of the cotton seedlings. When the concentration was increased to 10 or $20 \mu \mathrm{g} / \mathrm{ml}$, a negative effect on the growth of seedlings as measured by stem height, fresh weight, and dry weight was observed (Table 6).

\section{Influence of Fusaric Acid Concentrations on Cotton Verticillium Wilt}

The culture filtrates of isolate 10R-7 exhibited antifungal activity against $V$. dahliae, and the antifungal substance was deduced to be fusaric acid. We measured the ability of fusaric acid to control cotton Verticillium wilt in a greenhouse experiment. Fusaric acid was applied at different concentrations $(2.5,5,10,15,20,25$, 40 , and $80 \mu \mathrm{g} / \mathrm{ml})$. Fusaric acid did not significantly $(P>0.05)$ reduce the severity of the disease (Table 7). It may play a minor role in the suppression of the disease caused by $V$. dahliae.

\section{Colonization of Fusarium proliferatum 10R-7 on Cotton Roots}

In contrast to the wild-type isolate $10 \mathrm{R}-7$, isolate $10 \mathrm{R}$ 7GFP emitted green fluorescence in hyphae and conidia (Supplementary Figure 3). The 10R-7GFP-treated cotton seedlings were sampled and the taproots were observed under an Olympus IX81 for endophytic colonization by 10R-7GFP. While green fluorescence was not detected in the control group treated with water and the wild-type isolate 10R-7, green fluorescence was detected in the cotton root group inoculated with isolate 10R-7GFP. The conidia of 10R-7GFP attached to the surface of the cotton roots half an hour after inoculation with isolate 10R-7GFP. The conidia germinated, formed hyphae, and entered into the taproots at 1,2, and $7 \mathrm{dpi}$. The hyphae of 10R-7GFP were observed in the intercellular space and vascular bundles of the taproots of cotton seedlings at $1 \mathrm{dpi}$. At $7 \mathrm{dpi}$, the hyphae had colonized mainly along the axis of the taproots of cotton seedlings (Figure 7).

\section{DISCUSSION}

In the present research, 93 fungal isolates were obtained from surface-sterilized tissues of cotton grown in Hubei Province of central China. The results suggested that the endophytic fungi residing in cotton plants are highly diverse. These isolates were identified as belonging to 20 species according to morphological characteristics and ITS sequence identification, including Fusarium spp., Alternaria spp., Phomopsis spp., P. cucumerina, A. vagum, Diaporthe spp., Colletotrichum spp., B. dothidea, Bionectria ochroleuca, L. chartarum, Trichoderma spp., and others. Among these species, the most dominant were Fusarium spp. and Alternaria spp. Previous research reported 373 isolates from the endorhiza, rhizosphere, and bulk soil of cotton plants, and 12 fungal antagonists against $V$. dahliae were obtained (Zheng et al., 2011). Among these 12 fungal antagonists screened, five belonged to Fusarium spp., accounting for almost half of the 12 isolates. The results also showed that Fusarium spp. may be a dominant species of cotton endophytes. The community diversity of the endophytic fungi in cotton in this study was not very comprehensive due to the limitation of the samples, unculturable endophytic fungi, etc. Additional studies using extensive sampling strategies such as molecular sequencing technology are necessary to obtain more complete data for the endophytic fungal community in cotton. In addition 

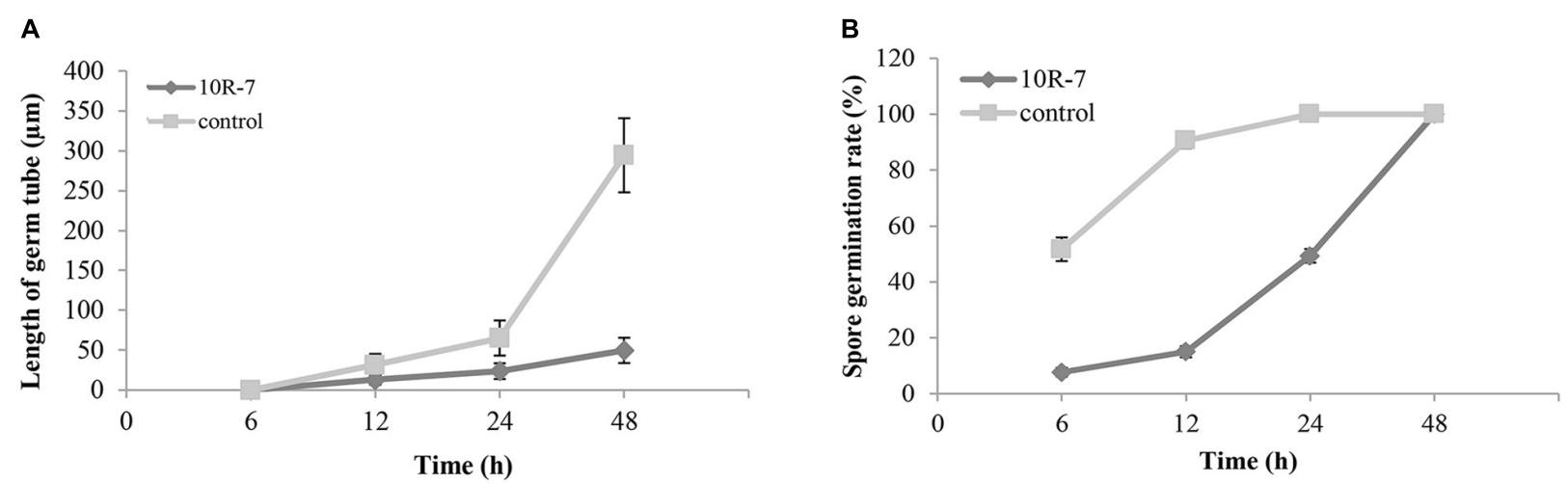

FIGURE 4 | Effect of culture filtrates of $F$. proliferatum 10R-7 (the concentration is 10\%) on germ tube elongation (A) and spore germination (B) of V. dahliae.

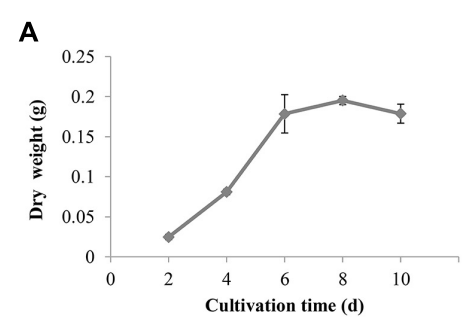

$\mathbf{B}$

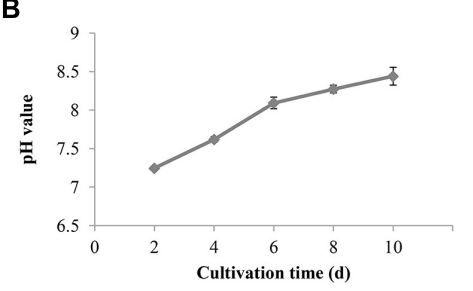

C
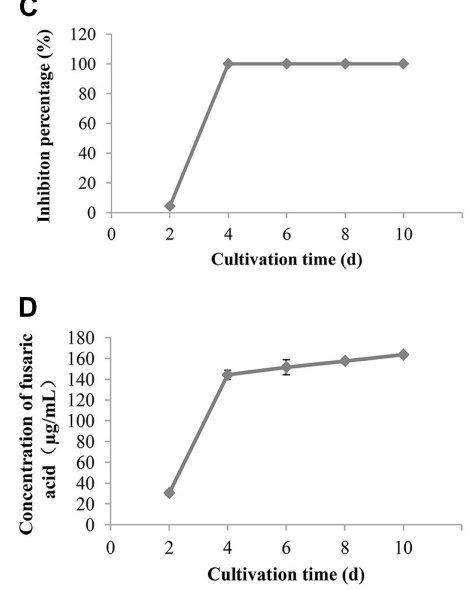

FIGURE 5 | The change of the biomass (A), pH value (B), inhibition rate (C), and content of fusaric acid (D) of the culture filtrates of $F$. proliferatum 10R-7 on V. dahliae at different culture times.

to studying the diversity of the endophytes in cotton, our other main objective was to screen potential BCAs against cotton Verticillium wilt.
Biological control has attracted increasing attention from researchers for suppressing soilborne diseases such as Verticillium wilt because of its environmental friendliness and pollution avoidance due to reduced pesticide use. However, the first step is to find a suitable source of potential BCAs. Previous studies indicated that endophytes were a rich source of BCAs. Huang et al. (2020) evaluated the capacity of endophytic fungi isolated from cucurbit plants to control soilborne diseases, and the results showed that endophytic fungi from cucurbits have great potential as biocontrol agents. The endophyte Cladorrhinum foecundissimum could colonize the roots of cotton to promote the absorption of phosphorus and plant growth (Gasoni and Stegman de Gurfinkel, 1997). Li et al. (2014) reported that a total of 642 endophytic fungi from 27 genera were isolated from the roots (141), stems (111), and leaves (391) of G. hirsutum plants. Thirty-nine isolates showed different degrees of antifungal activity against $V$. dahliae VD080. Narisawa et al. (2002) reported that a few fungal endophytes from eggplants, melon, tomato, strawberry, and Chinese cabbage are potential BCAs for Verticillium wilt of eggplant.

Moreover, the screening strategy is crucial. The traditional screening strategy is to evaluate the in vitro antagonism of screening strains toward $V$. dahliae in the first round of screening. However, some of the strains might be effective in controlling cotton Verticillium wilt while lacking in vitro antagonism. Therefore, we adopted the strategy of using greenhouse experiments for the first round of screening. First, we discarded those of the 93 isolates that did not produce spores. Then, the remaining 72 isolates were further screened according to screening in greenhouse experiments. The results revealed some potential biocontrol strains among these cotton endophytes. Five fungal antagonists were obtained according to multiple screening, and their average biocontrol efficiencies against cotton Verticillium wilt in the greenhouse experiment were higher than $50 \%$. The five most effective fungal antagonists screened in greenhouse experiments all belong to Fusarium spp. One possible reason is that the environment (temperature and soil) is suitable for Fusarium spp. to grow in central China and Fusarium spp. is a dominant genera in isolated endophytic fungi. The other possible reason is that it is related 


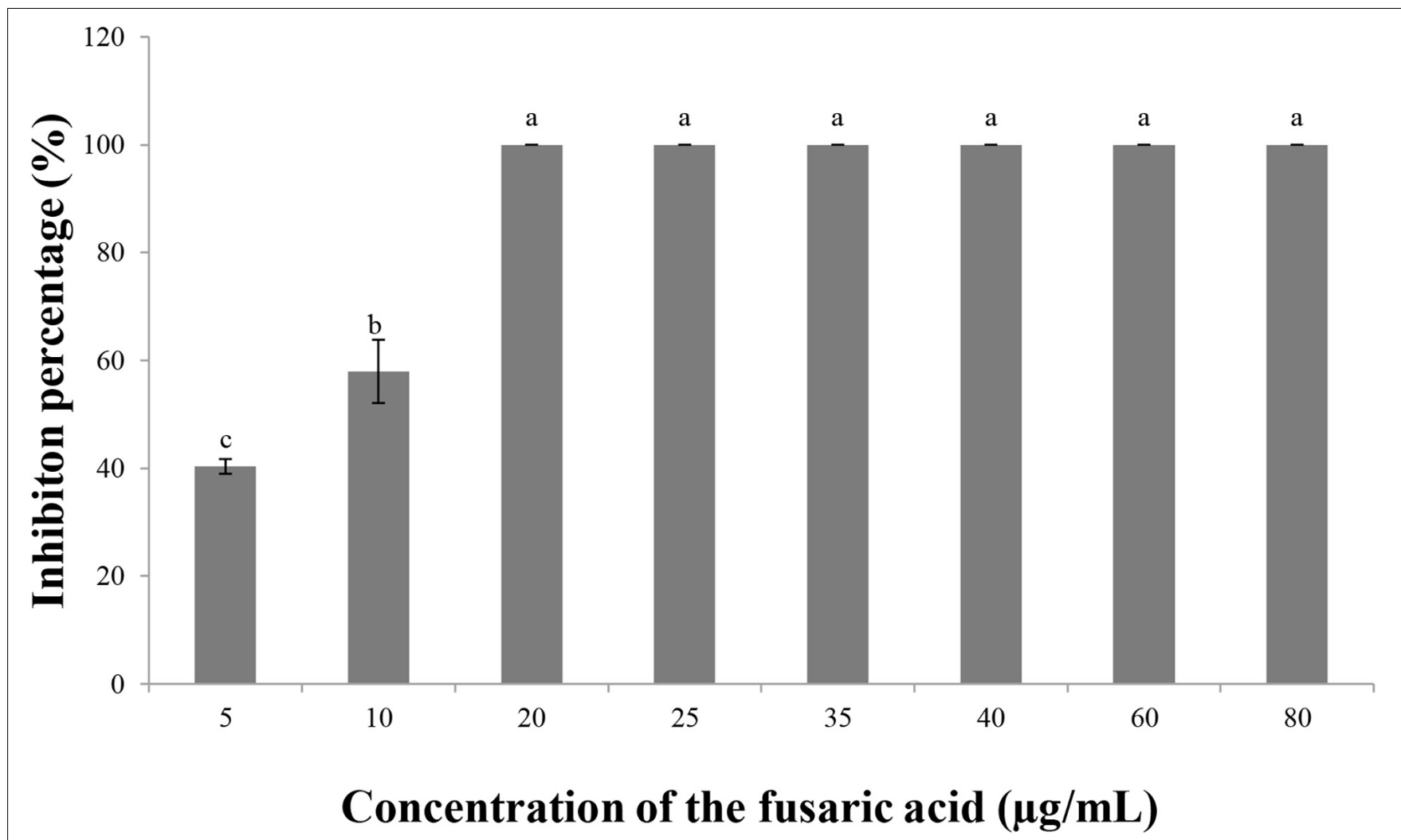

FIGURE 6 | Inhibitory effect of fusaric acid of different concentrations on $V$. dahliae.

TABLE 6 | Effects of the different concentrations of fusaric acid on growth index of cotton.

\begin{tabular}{|c|c|c|c|c|}
\hline Treatment & Stem height (cm) & Root length (cm) & Fresh weight (g) & Dry weight (g) \\
\hline Control & $15.2 \pm 2.2 \mathrm{a}^{1}$ & $9.1 \pm 2.3 \mathrm{a}^{1}$ & $2.5 \pm 0.7 \mathrm{a}^{1}$ & $0.2 \pm 0.1 \mathrm{a}^{1}$ \\
\hline Fusaric acid (5 $\mu \mathrm{g} / \mathrm{ml})$ & $14.2 \pm 1.7 \mathrm{ab}$ & $9.9 \pm 2.8 \mathrm{a}$ & $2.4 \pm 0.4 \mathrm{a}$ & $0.2 \pm 0.1 \mathrm{a}$ \\
\hline Fusaric acid (10 $\mu \mathrm{g} / \mathrm{ml})$ & $13.6 \pm 1.9 b$ & $8.8 \pm 1.6 \mathrm{a}$ & $2.0 \pm 0.4 b$ & $0.2 \pm 0.0 b$ \\
\hline Fusaric acid $(20 \mu \mathrm{g} / \mathrm{ml})$ & $13.4 \pm 1.2 b$ & $7.1 \pm 2.6 b$ & $2.0 \pm 0.6 b$ & $0.2 \pm 0.1 \mathrm{ab}$ \\
\hline
\end{tabular}

${ }^{1}$ Means \pm SE within each column in each trial followed by the same letters are not significantly different $(P>0.05)$ according to Duncan's multiple test.

to the screening method of greenhouse experiment used in research. In the screening experiment, the conidial suspension of $V$. dahliae mixed with endophytic fungi was inoculated with the cotton seedlings and Fusarium spp. have faster growth rate and stronger competitive ability than $V$. dahliae. Numerous studies have reported that nonpathogenic isolates of $F$. oxysporum have the potential to protect plants against pathogenic $F$. oxysporum and $V$. dahliae through competition for nutrients and space, induction of resistance responses, and production of antibiotic substances (Fravel et al., 2003; Pantelides et al., 2009; Zhang et al., 2015). These endophytes will be screened to be potential BCAs to suppress cotton Verticillium wilt in the future.

The most effective isolate 10R-7 screened in this study produced volatile organic compounds (VOCs) and secondary metabolism products that inhibit the growth of $V$. dahliae on agar media. The results showed that compared with the control treatment, $F$. proliferatum 10R-7 treatment significantly reduced wilt symptom development in cotton under greenhouse conditions. This is the first report about the biocontrol efficacy of the $F$. proliferatum against $V$. dahliae. Then, the biocontrol mechanisms of $F$. proliferatum 10R-7 were investigated in terms of competition for ecological sites and production of antibiotic substances. The inhibition percentage of VOCs that F. proliferatum 10R-7 produced was $69.71 \%$, and previous research reported that an endophyte F. oxysporum CanR-46 from oilseed rape produced VOCs that suppressed the growth of pathogens Botrytis cinerea and Sclerotinia sclerotiorum. GC-MS analysis identified a total of 19 main VOCs. Whether the VOCs that F. proliferatum 10R-7 produced were similar to the VOCs of F. oxysporum CanR-46 will be studied in the future.

The CFs of F. proliferatum 10R-7 shake-cultured in CDM for 7 days inhibited mycelial growth, spore germination, and mycelial elongation of $V$. dahliae. The CFs inhibited $V$. dahliae 
TABLE 7 | Effects of the different concentrations of fusaric acid on cotton Verticillium wilt in greenhouse experiment.

\begin{tabular}{|c|c|c|}
\hline Treatment & Disease incidence & Disease index \\
\hline \multicolumn{3}{|l|}{ Trial 1} \\
\hline V. dahliae alone & $86.1 \% \mathrm{a}^{1}$ & $66.7 \pm 15.7 \mathrm{a}^{1}$ \\
\hline $2.5 \mu \mathrm{g} / \mathrm{ml}$ fusaric acid $+V$. dahliae & $77.0 \%$ a & $56.9 \pm 22.9 a$ \\
\hline $5 \mu \mathrm{g} / \mathrm{ml}$ fusaric acid $+V$. dahliae & $77.8 \%$ a & $61.9 \pm 8.6 \mathrm{a}$ \\
\hline $15 \mu \mathrm{g} / \mathrm{ml}$ fusaric acid $+V$. dahliae & $72.2 \%$ a & $57.6 \pm 10.7 \mathrm{a}$ \\
\hline $25 \mu \mathrm{g} / \mathrm{ml}$ fusaric acid $+V$. dahliae & $65.8 \%$ a & $50.6 \pm 26.0 \mathrm{a}$ \\
\hline \multicolumn{3}{|l|}{ Trial 2} \\
\hline V. dahliae alone & $100.0 \% \mathrm{a}^{1}$ & $90.0 \pm 5.0 \mathrm{a}$ \\
\hline $10 \mu \mathrm{g} / \mathrm{ml}$ fusaric acid $+V$. dahliae & $86.7 \%$ b & $80.8 \pm 19.1 \mathrm{a}$ \\
\hline $20 \mu \mathrm{g} / \mathrm{ml}$ fusaric acid $+V$. dahliae & $96.7 \% \mathrm{ab}$ & $74.2 \pm 12.3 \mathrm{a}$ \\
\hline $40 \mu \mathrm{g} / \mathrm{ml}$ fusaric acid $+V$. dahliae & $100.0 \% \mathrm{a}$ & $83.3 \pm 2.9 \mathrm{a}$ \\
\hline \multicolumn{3}{|l|}{ Trial 3} \\
\hline V. dahliae alone & $97.8 \% a^{1}$ & $73.3 \pm 11.6 \mathrm{a}$ \\
\hline $20 \mu \mathrm{g} / \mathrm{ml}$ fusaric acid $+V$. dahliae & $78.9 \% \mathrm{ab}$ & $56.4 \pm 11.0 \mathrm{a}$ \\
\hline $40 \mu \mathrm{g} / \mathrm{ml}$ fusaric acid $+V$. dahliae & $77.1 \% \mathrm{ab}$ & $51.9 \pm 13.8 a$ \\
\hline $80 \mu \mathrm{g} / \mathrm{ml}$ fusaric acid $+V$. dahliae & $68.9 \% \mathrm{~b}$ & $46.1 \pm 21.8 \mathrm{a}$ \\
\hline
\end{tabular}

${ }^{1}$ Means \pm SE within each column in each trial followed by the same letters are not significantly different $(P>0.05)$ according to Duncan's multiple test.

growth, and the inhibition rate of $4 \%$ CFs reached $100 \%$ on agar media. Analysis of the CFs of F. proliferatum 10R-7 shakecultured in CDM showed that the main antifungal substance was fusaric acid, and the results showed that fusaric acid also inhibited $V$. dahliae growth. The inhibition rate of $20 \mu \mathrm{g} / \mathrm{ml}$ fusaric acid reached $100 \%$ on agar media. However, as a type of Fusarium toxin, fusaric acid had a negative impact on plant growth and caused the wilting of plants at high concentrations. It was confirmed that 10 and $20 \mu \mathrm{g} / \mathrm{ml}$ fusaric acid had negative impacts on plant growth. However, when cotton roots were inoculated with F. proliferatum 10R-7, fusaric acid was not produced in the root exudates (data not shown). Fusaric acid was also not produced when F. proliferatum $10 \mathrm{R}-7$ was shake-cultured in PDB medium. The results indicated that $F$. proliferatum 10R-7 produced fusaric acid under certain conditions. A high concentration of fusaric acid could produce a negative effect and cause the wilting of plants, and concentrations of fusaric acid below a certain level did not cause inhibition. Therefore, pure fusaric acid did not play a good biocontrol role. When a spore suspension of $V$. dahliae mixed with different concentrations of fusaric acid was inoculated, the development of Verticillium wilt symptoms in cotton did not significantly decrease. It seemed that the production of fusaric acid had a minor correlation with the biocontrol effect of F. proliferatum 10R-7 against $V$. dahliae. The production of fusaric acid was verified to not be the main factor in the biocontrol mechanism of $F$. proliferatum 10R-7. Therefore, we analyzed another category: competition for space.

For this purpose, we transformed F. proliferatum 10R-7 with a GFP gene. GFP transformation has become a common method for detection in plant-pathogen interaction studies. GFP-labeled F. proliferatum 10R-7 showed the same characteristics as the wild-type strain in morphology, growth rates, and control effects for $V$. dahliae in the greenhouse experiments (Supplementary Tables 2, 3 and Supplementary Figure 3). Previous research has shown dynamic changes in $V$. dahliae at the root after the cotton roots had been inoculated. At $1 \mathrm{dpi}$, the conidia on root surfaces germinated and formed hyphal networks along the cell junctions (Pantelides et al., 2009; Zhao et al., 2014). By approximately $7 \mathrm{dpi}, V$. dahliae had penetrated and massively colonized the xylem vessels of the roots. It seems that F. proliferatum 10R-7 entered the root and dominated the same ecological niche when the roots were inoculated with the spore suspension of GFP-labeled F. proliferatum 10R-7. This could result from competition for space, one of the main
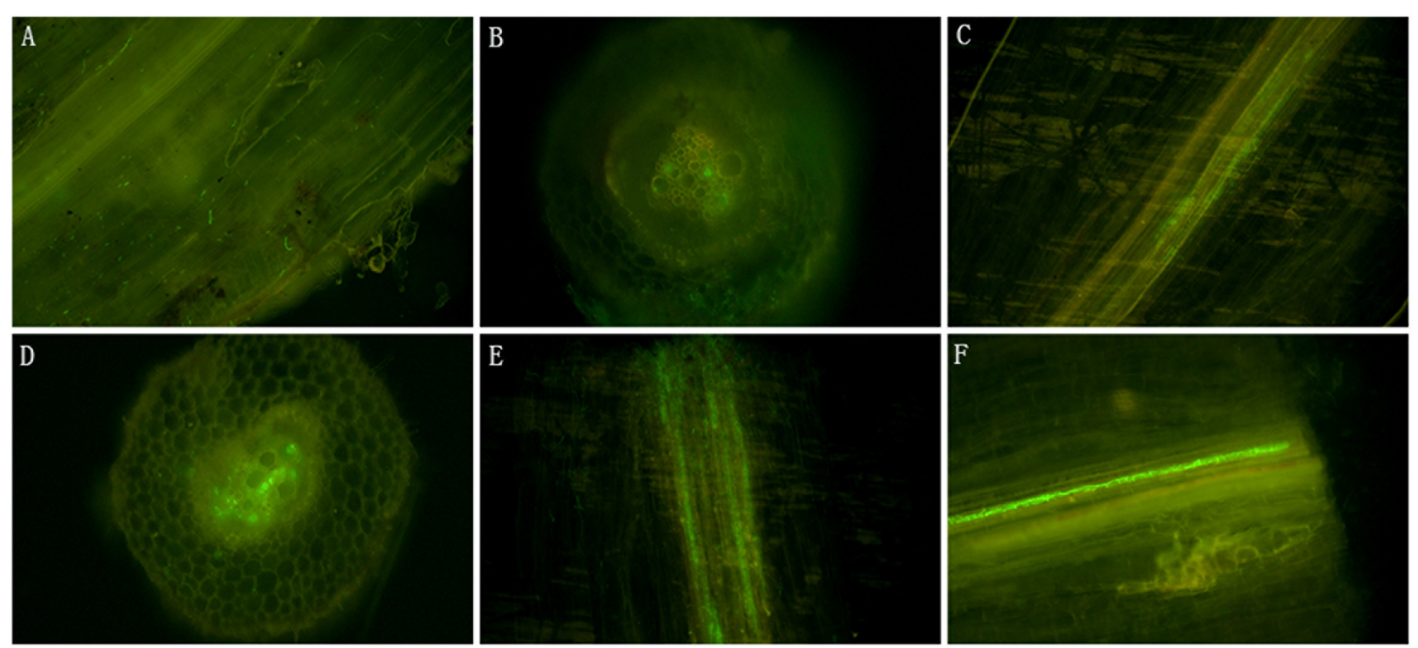

FIGURE 7 | The microscopic micrographs showing endophytic colonization of cotton roots by Fusarium proliferatum 10R-7GFP. (A) Longitudinal sections of the roots inoculated with F. proliferatum 10R-7GFP $\left(25^{\circ} \mathrm{C}\right.$, half past hour postinoculation); (B,C) longitudinal and cross sections of the roots inoculated with F. proliferatum 10R-7GFP at the first day postinoculation, respectively; (D,E) longitudinal and cross sections of the roots inoculated with F. proliferatum 10R-7GFP at the second day postinoculation, respectively. (F) Longitudinal sections of the roots inoculated with F. proliferatum 10R-7GFP at the seventh day postinoculation. 
mechanisms of action of BCAs against pathogens (Pantelides et al., 2009; Gizi et al., 2011). Meanwhile, when the cotton roots were inoculated with different concentrations $\left(1 \times 10^{4}\right.$, $1 \times 10^{5}, 1 \times 10^{6}$, and $1 \times 10^{7}$ conidia/ml) of $F$. proliferatum 10R-7 spore solutions before being inoculated with a spore suspension of $V$. dahliae, the biocontrol efficacy increased as the spore concentration increased, and the biocontrol efficacy of $1 \times 10^{7}$ conidia/ml of $F$. proliferatum $10 \mathrm{R}-7$ spore solutions was greater than those of the other treatments. This result also indicated that when the inoculation spore concentrations increased, F. proliferatum 10R-7 dominated the ecological niche to a greater degree to prevent the invasion of the pathogen $V$. dahliae.

Analysis of the biocontrol mechanism of BCAs will help us to improve control strategies. Another important problem is the safety of BCAs. In the present study, two inoculation methods, the mixed inoculation with a spore suspension of F. proliferatum 10R-7 and $V$. dahliae and the inoculation with a spore suspension of $F$. proliferatum 10R-7 before inoculation with $V$. dahliae, could both reduce the symptom development of Verticillium wilt in cotton significantly in greenhouse experiments. However, the toxic substance fusaric acid could be produced in the CFs of F. proliferatum 10R7 shake-cultured in Czapek-Dox medium. The production of the toxin means that the application of F. proliferatum $10 \mathrm{R}-7$ poses some risk to some other plants. Therefore, future studies will focus on how to avoid the negative effect when taking full advantage of BCAs.

Overall, we investigated two possible mechanisms of action of $F$. proliferatum 10R-7 against $V$. dahliae: the production of antifungal substances and the competition for space. Some previous research has revealed the biocontrol mechanism of nonpathogenic $F$. oxysporum against $V$. dahliae. However, to our knowledge, this is the first study on the biocontrol mechanism of $F$. proliferatum against $V$. dahliae. Our results showed that competition for space on the root surface may be the main biocontrol mechanism that F. proliferatum 10R7 uses against $V$. dahliae. The production of secondary metabolites was not considered to be the biocontrol mechanisms of F. proliferatum 10R-7 against $V$. dahliae. Promoting the application of $F$. proliferatum $10 \mathrm{R}-7$ as a promising BCA warrants further study.

\section{CONCLUSION}

Ninety-three endophytic fungal strains were isolated from healthy cotton samples: 23 strains from the roots and 70 strains from the stems. These strains were identified as belonging to 20 species according to morphological characterization and ITS sequence analysis. Fusarium and Alternaria were the two dominant genera, constituting $19.4 \%$ of the total strains. The diversity of the cotton endophytes was analyzed. Then, 72 spore-producing strains were tested for the suppression of CVW caused by $V$. dahliae in a greenhouse. Five strains exhibited effective suppression of CVW with average efficacy values higher than $50 \%$. As one of the effective strains, the possible biocontrol mechanisms of $F$. proliferatum 10R-7 against $V$. dahliae were investigated by identifying the antifungal metabolites of F. proliferatum $10 \mathrm{R}-7$ and assessing its colonization in cotton roots. The results showed that endophytic colonization may play a role in the suppression of infection of cotton by V. dahliae.

\section{DATA AVAILABILITY STATEMENT}

The original contributions presented in the study are included in the article/Supplementary Material, further inquiries can be directed to the corresponding author/s.

\section{AUTHOR CONTRIBUTIONS}

LJ, LY, GL, and PW conceived and designed the experiments. LJ, WL, and DX performed the experiments. LJ and NY organized and analyzed the data. LJ, PW, and GL wrote the manuscript. GL supervised the study. All authors read and approved the final manuscript.

\section{FUNDING}

This work was supported by the National Special Transgenic Project of China (No. 2016ZX08011-002) and the National Key Research and Development Program (2017YFD0201907).

\section{SUPPLEMENTARY MATERIAL}

The Supplementary Material for this article can be found online at: https://www.frontiersin.org/articles/10.3389/fmicb.2021. 698930/full\#supplementary-material

Supplementary Figure 1 | HPLC and TIC comparison and analysis of the culture filtrates of F. proliferatum 10R-7 and fusaric acid. (A) HPLC chromatography and TIC (total ion chromatogram) of the culture filtrates of F. proliferatum 10R-7. (B) HPLC chromatography and TIC of fusaric acid. (C) UV absorption spectrogram of the culture filtrates of $F$. proliferatum 10R-7. (D) UV absorption spectrogram of fusaric acid.

Supplementary Figure $2 \mid \mathrm{TIC}$ and UV absorption spectrogram of the culture filtrates of $F$. proliferatum 10R-7 in PDB medium and CDM (Czapek-Dox medium). (A) TIC of the culture filtrates of $F$. proliferatum 10R-7 in PDB medium. (B) UV absorption spectrogram of the culture filtrates of $F$. proliferatum 10R-7 in PDB medium. (C) TIC of the culture filtrates of $F$. proliferatum 10R-7 in CDM. (D) UV absorption spectrogram of the culture filtrates of $F$. proliferatum 10R-7 in CDM.

Supplementary Figure 3 | The morphological characteristics of $F$. proliferatum 10R-7 and F. proliferatum 10R-7GFP. (A,B) The colony morphology of F. proliferatum 10R-7 and F. proliferatum 10R-7GFP, respectively. (C,D) Mycelial and conidial morphology of the proliferatum 10R-7GFP.

Supplementary Table 1| The preliminary screening of the endophytes against Verticillium dahliae.

Supplementary Table 2 | The comparison of growth rates of the two isolates F. proliferatum 10R-7 and F. proliferatum 10R-7GFP.

Supplementary Table $\mathbf{3} \mid$ Control effect of $F$. proliferatum 10R-7GFP on suppression of cotton Verticillium wilt caused by $V$. dahliae in greenhouse. 


\section{REFERENCES}

Aguado, A., Santos, B. D. L., Blanco, C., and Romero, F. (2008). Study of gene effects for cotton yield and Verticillium wilt tolerance in cotton plant (Gossypium hirsutum L.). Field Crop Res. 107, 78-86. doi: 10.1016/j.fcr.2007.12. 018

Angelopoulou, D. J., Naska, E. J., Paplomatas, E. J., and Tjamos, S. E. (2014). Biological control agents (BCAs) of Verticillium wilt: influence of application rates and delivery method on plant protection, triggering of host defence mechanisms and rhizosphere populations of BCAs. Plant Pathol. 63, 1062 1069. doi: 10.1111/ppa.12198

Backman, P. A., and Sikora, R. A. (2008). Endophytes: an emerging tool for biological control. Biol. Control 46, 1-3. doi: 10.1016/j.biocontrol.2008. 03.009

Berg, G., Fritze, A., Roskot, N., and Smalla, K. (2001). Evaluation of potential biocontrol rhizobacteria from different host plants of Verticillium dahliae Kleb. J. Appl. Microbiol. 91, 963-971. doi: 10.1046/j.1365-2672.2001. 01462.x

Berg, G., Zachow, C., Lottmann, J., Gutz, M., Costa, R., and Smalla, K. (2005). Impact of plant species and site on rhizosphere-associated fungi antagonistic to Verticillium dahliae Kleb. Appl. Environ. Microbiol. 71, 4203-4213. doi: 10. 1128/AEM.71.8.4203-4213.2005

Deketelaere, S., Tyvaert, L., França, S. C., and Höfte, M. (2017). Desirable traits of a good biocontrol agent against Verticillium wilt. Front. Microbiol. 8:1186. doi: $10.3389 /$ fmicb. 2017.01186

Díaz, J., Silvar, C., Varela, M. M., Bernal, A., and Merino, F. (2005). Fusarium confers protection against several mycelial pathogens of pepper plants. Plant Pathol. 54, 773-780. doi: 10.1111/j.1365-3059.2005. 01285.x

Dutta, B. K. (1981). Studies on some fungi isolated from the rhizosphere of tomato plants and the consequent prospect for the control of Verticillium wilt. Plant Soil 63, 209-216. doi: 10.1007/BF02374599

Ek-Ramos, M. J., Zhou, W. Q., Valencia, C. U., Antwi, J. B., Kalns, L. L., Morgan, G. D., et al. (2013). Spatial and temporal variation in fungal endophyte communities isolated from cultivated cotton(Gossypium hirsutum). PLoS One 8:e66049. doi: 10.1371/journal.pone.0066049

Erdogan, O., and Benlioglu, K. (2010). Biological control of Verticillium wilt on cotton by the use of fluorescent Pseudomonas spp. under field conditions. Biol. Control 153, 39-45. doi: 10.1016/j.biocontrol.2009. 11.011

Fravel, D., Olivain, C., and Alabouvette, C. (2003). Fusarium oxysporum and its biocontrol. New Phytol. 157, 493-502. doi: 10.1046/j.1469-8137.2003. 00700.x

García, M., Arriagada, C., García-Romera, I., and Ocampo, J. A. (2011). Are plant cell wall hydrolysing enzymesof saprobe fungi implicated in the biological control of the Verticillium dahliae pathogensis? Crop Prot. 30, 85-87. doi: 10.1016/j.cropro.2010.09.007

Gasoni, L., and Stegman de Gurfinkel, B. (1997). The endophyte Cladorrhinum foecundissimum in cotton roots, effects on phosphorus uptake and host growth. Mycological Research 101, 867-870. doi: 10.1017/S0953756296003462

Gizi, D., Stringlis, I. A., Tjamos, S. E., and Paplomatas, E. J. (2011). Seedling vaccination by stem injecting a conidial suspension of F2, a non-pathogenic Fusarium oxysporum strain, suppresses Verticillium wilt of eggplant. Biol. Control 58, 387-392. doi: 10.1016/j.biocontrol.2011.06.009

Hallmann, J., Berg, G., and Schulz, B. (2006). "Isolation procedures for endophytic microorganisms," in Microbial Root Endophytes, eds B. J. E. Schulz, C. J. C. Boyle, and T. N. Sieber (Berlin: Springer), 299-319. doi: 10.1007/3-540-33526$9 \_17$

Hassani, M. A., Duran, P., and Hacquard, S. (2018). Microbial interactions within the plant holobiont. Microbiome 6:58. doi: 10.1186/s40168-0180445-0

Huang, L. Q., Niu, Y. C., Su, L., Deng, H., and Lyu, H. (2020). The potential of endophytic fungi isolated from cucurbit plants for biocontrol of soilborne fungal diseases of cucumber. Microbiol. Res. 231:126369. doi: 10.1016/j.micres. 2019.126369

Kirk, P. M., Cannon, P. F., and Minter, D. W. (2008). In Dictionary of the Fungi, 10th Edn. Wallingford: CAB International.
Klosterman, S. J., Atallah, Z. K., Vallad, G. E., and Subbarao, K. V. (2009). Diversity, pathogenicity, and management of Verticillium species. Annu. Rev. Phytopathol. 47, 39-62. doi: 10.1146/annurev-phyto-080508081748

Larkin, R. P., and Fravel, D. R. (1999). Mechanisms of action and dose-response relationships governing biological control of fusarium wilt of tomato by nonpathogenic Fusarium spp. Phytopathology 89, 1152-1161. doi: 10.1094/ PHYTO.1999.89.12.1152

Li, Z. F., Wang, L. F., Feng, Z. L., Zhao, L. H., and Zhu, H. Q. (2014). Diversity of endophytic fungi from different Verticillium wilt-resistant Gossypium hirsutum and evaluation of antifungal activity against Verticillium dahliae in vitro. J. Microbiol. Biotechnol. 24, 1149-1161. doi: 10.4014/jmb.1402. 02035

Lyu, A., Liu, H., Che, H., Yang, L., Zhang, J., Wu, M., et al. (2017). Reveromycins A and B from streptomyces sp. 3-10: antifungal activity against plant pathogenic fungi in vitro and in a strawberry food model system. Front. Microbiol. 8:550. doi: $10.3389 /$ fmicb. 2017.00550

Ma, C., Jian, G., and Zheng, C. (2002). Advance in cotton breeding for resistance to Fusarium and Verticillium wilt in the last fifty years in China. Agric. Sci. China $35,508-513$.

Malandraki, I., Tjamos, S. E., Pantelides, I. S., and Paplomatas, E. J. (2008). Thermal inactivation of compost suppressiveness implicates possible biological factors in disease management. Biol. Control 44, 180-187. doi: 10.1016/j.biocontrol.2007. 10.006

Mercado-Blanco, J., Rodriguez-Jurado, D., Hervas, A., and Jimenez-Diaz, R. M. (2004). Suppression of Verticillium wilt in olive planting stocks by rootassociated fluorescent Pseudomonas spp. Biol. Control 30, 474-486. doi: 10. 1016/j.biocontrol.2004.02.002

Nagtzaam, M. P. M., Bollen, G. J., and Termorshuizen, A. J. (1998). Efficacy of Talaromyces flavus alone or in combination with other antagonists in controlling Verticillium dahliae in growth chamber experiments. J. Phytopathol. 146, 165-173. doi: 10.1111/j.1439-0434.1998. tb04674.x

Narisawa, K., Kawamata, H., Currah, R. S., and Hashiba, T. (2002). Suppression of Verticillium Wilt in eggplant by some fungal root endophytes. Eur. J. Plant Pathol. 108, 103-109. doi: 10.1023/A:1015080311041

Olivain, C., Humbert, C., Nahalkova, J., Fatehi, J., and Alabouvette, C. (2006). Colonization of tomato root by pathogenic and nonpathogenic Fusarium oxysporum strains inoculated together and separately into the soil. Appl. Environ. Microbiol. 72, 1523-1531. doi: 10.1128/AEM.72.2.1523-1531. 2006

Pantelides, I. S., Tjamos, S. E., Striglis, I. A., Chatzipavlidis, I., and Paplomatas, E. J. (2009). Mode of action of a non-pathogenic Fusarium oxysporum strain against Verticillium dahliae using real time QPCR analysis and biomarker transformation. Biol. Control 50, 30-36. doi: 10.1016/j.biocontrol.2009.01.010

Potshangbam, M., Devi, S. I., Sahoo, D., and Strobel, G. A. (2017). Functional characterization of endophytic fungal community associated with Oryza sativa L. and Zea mays L. Front. Microbiol. 8:325. doi: 10.3389/fmicb.2017.00325

Rodriguez, R. J., White, J. F. Jr., Arnold, A. E., and Redman, R. S. (2009). Fungal endophytes:diversity and functional roles. New Phytol. 182, 314-330. doi: 10. 1111/j.1469-8137.2009.02773.x

Shi, L., Wang, B., and Wen, X. (1993). Study on strain of defoliating type of Verticillium Wilt in cotton. Cotton Sci. 1, 89-92.

Uppal, A. K., Hadrami, A. E., Adam, L. R., Tenuta, M., and Daayf, F. (2008). Biological control of potato Verticillium wilt under controlled and field conditions using selected bacterial antagonists and plant extracts. Biol. Control 44, 90-100. doi: 10.1016/j.biocontrol.2007. 10.020

Varo, A., Moral, J., Lozano-Tóvar, M. D., and Trapero, A. (2016a). Development and validation of an inoculation method to assess the efficacy of biological treatments against Verticillium wilt in olive trees. Biol. Control 61, 283-292. doi: 10.1007/s10526-015-9710-3

Varo, A., Raya-Ortega, M. C., and Trapero, A. (2016b). Selection and evaluation of micro-organisms for biocontrol of Verticillium dahliae in olive. J. Appl. Microbiol. 121, 767-777. doi: 10.1111/jam.13199

Veloso, J., and Díaz, J. (2012). Fusarium oxysporum Fo47 confers protection to pepper plants against Verticillium dahliae and Phytophthora capsici, and induces 
the expression of defence genes. Plant Pathol. 61, 281-288. doi: 10.1111/j.13653059.2011.02516.x

Zhang, Q., Yang, L., Zhang, J., Wu, M., Chen, W., Jiang, D., et al. (2015). Production of anti-fungal volatiles by non-pathogenic Fusarium oxysporum and its efficacy in suppression of Verticillium wilt of cotton. Plant Soil 392, 101-114. doi: 10.1007/s11104-015-2448-y

Zhang, Q., Zhang, J., Yang, L., Zhang, L., Jiang, D., Chen, W., et al. (2014). Diversity and biocontrol potential of endophytic fungi in Brassica napus. Biol. Control 72, 98-108. doi: 10.1016/j.biocontrol.2014.02.018

Zhao, P., Zhao, Y. L., Jin, Y., Zhang, T., and Guo, H. S. (2014). Colonization process of Arabidopsis thaliana roots by a green fluorescent protein-tagged isolate of Verticillium dahliae. Protein Cell 5, 94-98. doi: 10.1007/s11104-015$2448-\mathrm{y}$

Zheng, Y., Xue, Q. Y., Xu, L. L., Xu, Q., Lu, S., Gu, C., et al. (2011). A screening strategy of fungal biocontrol agents towards Verticillium wilt of cotton. Biol. Control 56, 209-216. doi: 10.1016/j.biocontrol.2010.11.010
Conflict of Interest: The authors declare that the research was conducted in the absence of any commercial or financial relationships that could be construed as a potential conflict of interest.

Publisher's Note: All claims expressed in this article are solely those of the authors and do not necessarily represent those of their affiliated organizations, or those of the publisher, the editors and the reviewers. Any product that may be evaluated in this article, or claim that may be made by its manufacturer, is not guaranteed or endorsed by the publisher.

Copyright $\odot 2021 \mathrm{Jin}$, Yang, Li, Xu, Yang, Li and Wan. This is an open-access article distributed under the terms of the Creative Commons Attribution License (CC BY). The use, distribution or reproduction in other forums is permitted, provided the original author(s) and the copyright owner(s) are credited and that the original publication in this journal is cited, in accordance with accepted academic practice. No use, distribution or reproduction is permitted which does not comply with these terms. 